\title{
Studies of the spectral and angular distributions of transition radiation using a silicon pixel sensor on a Timepix3 chip
}

\author{
J. Alozy ${ }^{a}$, N. Belyaev ${ }^{\text {b }}$, M. Campbell ${ }^{\text {a }}$, M. Cherry ${ }^{\mathrm{c}}$, F. Dachs ${ }^{\mathrm{a}, \mathrm{d}}$, S. Doronin ${ }^{\mathrm{b}}$, K. Filippov ${ }^{\mathrm{b}}$, \\ P. Fusco ${ }^{\text {eff }}$, F. Gargano ${ }^{\text {f }}$, E.H.M. Heijne ${ }^{\mathrm{n}}$, S. Konovalov ${ }^{g}$, D. Krasnopevtsev ${ }^{\text {b }}$, X. Llopart ${ }^{\mathrm{a}}$, \\ F. Loparco e,f , V. Mascagna ${ }^{\text {h,i }}$, M.N. Mazziotta ${ }^{\mathrm{f}}$, H. Pernegger ${ }^{\mathrm{a}}$, D. Ponomarenko ${ }^{\mathrm{b}}$, C. Rembser ${ }^{\mathrm{a}}$, \\ A. Romaniouk ${ }^{\text {b,* }}$, A.A. Savchenko ${ }^{\mathrm{b}, \mathrm{j}, 1}$, D. Schaefer ${ }^{\mathrm{k}}$, E.J. Schioppa ${ }^{\mathrm{a}}$, D.Yu. Sergeeva ${ }^{\mathrm{b}, \mathrm{j}, \mathrm{l}}$, \\ E. Shulga ${ }^{\text {b }}$, S. Smirnov ${ }^{\text {b }}$, Y. Smirnov ${ }^{\text {b }}$, M. Soldani ${ }^{\text {h,i }}$, P. Spinelli ${ }^{\text {e,f }}$, M. Strikhanov ${ }^{\text {b }}$, P. Teterin ${ }^{\text {b }}$, \\ V. Tikhomirov $^{\mathrm{g}}$, A.A. Tishchenko ${ }^{\mathrm{b}, \mathrm{j}, \mathrm{l}}$, M. van Beuzekom ${ }^{\mathrm{m}}$, B. van der Heijden ${ }^{\mathrm{m}}$, K. Vorobev $^{\mathrm{b}}$, \\ K. Zhukov ${ }^{g}$
}

a CERN, the European Organization for Nuclear Research, Esplanade des Particules 1, 1211 Geneva, Switzerland

${ }^{\mathrm{b}}$ National Research Nuclear University MEPhI (Moscow Engineering Physics Institute), Kashirskoe highway 31, Moscow, 115409, Russia

${ }^{\mathrm{c}}$ Louisiana State University, Baton Rouge, LA 70803, USA

'Technical University of Vienna, Karlsplatz 13, 1040 Vienna, Austria

e Dipartimento di Fisica “M. Merlin" dell'Università e del Politecnico di Bari, Via G. Amendola 173, 70126 Bari, Italy

${ }^{\mathrm{f}}$ Istituto Nazionale di Fisica Nucleare, Sezione di Bari, Via E. Orabona 4, 70126 Bari, Italy

${ }^{g}$ P. N. Lebedev Physical Institute of the Russian Academy of Sciences, Leninsky prospect 53, Moscow, 119991, Russia

${ }^{\text {h } I N F N ~ M i l a n o ~ B i c o c c a, ~ P i a z z a ~ d e l l a ~ S c i e n z a ~ 3, ~} 20126$ Milano, Italy

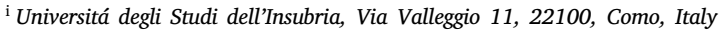

${ }^{\mathrm{j}}$ National Research Center "Kurchatov Institute", Akademika Kurchatova pl. 1, Moscow, Russia

${ }^{\mathrm{k}}$ University of Chicago, $5801 \mathrm{~S}$ Ellis Ave, Chicago, IL 60637, USA

${ }^{1}$ Belgorod National Research University, 85, Pobedy St., Belgorod, 308015, Russia

m Nikhef, Science Park 105, 1098 XG Amsterdam, The Netherlands

${ }^{\mathrm{n}}$ Institute for Experimental and Applied Physics of the Czech Technical University in Prague, Husova 2450/5, 11000, Prague 1, Czech Republic

\section{A R T I C L E I N F O}

\section{Keywords:}

Transition radiation detectors

Particle identification

Timepix3

Transition radiation

Pixel detectors

\begin{abstract}
A B S T R A C T
X-ray transition radiation detectors (TRDs) are used for particle identification in both high energy physics and astroparticle physics. In most of the detectors, emission of the X-ray transition radiation (TR) starts at Lorentz factors above $\gamma \sim 500$ and reaches saturation at $\gamma \sim 2 \div 3 \cdot 10^{3}$. However, many experiments require particle identification up to $\gamma \sim 10^{5}$, which is very difficult to achieve with conventional detectors. Semiconductor pixel detectors offer a unique opportunity for precise simultaneous measurements of spectral and angular parameters of TR photons. Test beam studies of the energy and the angular distributions of TR photons emitted by electrons and muons of different momenta crossing several types of radiators were performed at the CERN SPS with a $480 \mu \mathrm{m}$ thick silicon detector bonded to a Timepix 3 chip. High resolution images of the energy-angle phase space of the TR produced by different radiators were obtained and compared with MC simulations. The characteristic interference patterns are in agreement with the theoretical models with an unprecedented level of details. The studies presented in this paper also show that simultaneous measurements of both the energy and the emission angles of the TR X-rays could be used to enhance the particle identification performances of TRDs.
\end{abstract}

\section{Introduction}

Particle identification (PID) is one of the most important tasks of the detectors in high-energy physics (HEP) experiments. The technique based on X-ray transition radiation (TR) production is one of four types of non destructive methods of particle identification and covers the
Lorentz gamma factor range above $\sim 500$. The other three methods are based respectively on the time-of-flight (TOF), ionization (e.g. in Time Projection Chambers) and Cherenkov radiation effects and cover the Lorentz gamma factor $(\gamma)$ range below this value. Transition radiation X-rays are produced when a highly relativistic particle $(\gamma \gtrsim 500)$ crosses

\footnotetext{
* Corresponding author.

E-mail address: anatoli.romaniouk@cern.ch (A. Romaniouk).
} 
the interface between materials of different refractive index. Since the TR yield per boundary crossing is low, radiators which have multiple interface crossings (e.g., a set of foils) are used in real detectors. The $\mathrm{X}$-rays, ranging from a few $\mathrm{keV}$ to a few dozen $\mathrm{keV}$, are emitted in a forward direction at small angles (within a few mrad) with respect to the particle trajectory. In this energy range the TR exhibits a threshold effect as a function of the gamma factor [1,2]. The effective TR photon emission starts at a Lorentz factor of $\gamma_{t h r} \sim \ell_{1} \omega_{1} / c$ and saturates at $\gamma_{\text {sat }} \sim 0.6 \omega_{1} \sqrt{\ell_{1} \ell_{2}} / c$ [3], where $\omega_{1}$ is the radiator material plasma frequency, $\ell_{1}$ is its thickness, $\ell_{2}$ - the spacing between material elements and $\mathrm{c}$ - the speed of light. Interference effects in the radiators based on regularly spaced foils (with spacing from a fraction of $\mathrm{mm}$ to a few $\mathrm{mm}$ ) allow to obtain a coherent sum of amplitudes from different foils and enhance the TR yield (see for instance [3]). Interference effects are $\gamma$-dependent and change the angular distribution of TR photons with respect to that of a single foil which has a $1 / \gamma$ dependence. This phenomenon can be used to increase most probable TR production angle and allows a better separation of TR photons from the particle track.

Transition radiation detectors are widely used for electron-hadron separation in both accelerator and cosmic-ray experiments (see reviews [4-6]). Such detectors efficiently separate electrons (positrons) from pions with momenta up to more than $100 \mathrm{GeV} / \mathrm{c}$ and from protons in a momentum range of up to $\sim 1 \mathrm{TeV} / \mathrm{c}$. Hadrons have relatively small mass difference and their separation is a more difficult task. In the past the TRD technique was used to separate pions from protons and kaons at momenta of up to $200 \mathrm{GeV} / \mathrm{c}$ [7-10]. With growing energies of particles at modern or planned particle accelerator experiments as well as cosmic-ray experiments, the identification of particles with Lorentz factors $\gamma \sim 10^{5}$ is required [11-13]. A planned experiment at LHC [11] to measure the inclusive cross sections in the forward region for the production of charged particles in proton-proton, proton-nucleus and nucleus-nucleus interactions is a good example of such a need. In these collisions, secondary hadrons (mainly pions, kaons, protons) are produced with momenta of up to $6 \mathrm{TeV}$. Hadron identification at these energies is highly challenging since all particles have $1-\beta<10^{-5}$, and TRD is the only technique which can be used.

A typical TRD consists of a radiator followed by an X-ray detector. In conventional TRDs, PID is performed exploiting information about the energy losses in the detectors (usually gaseous detectors), which include the ionizing particle energy losses and the energies of the absorbed TR photons. This approach is proven to be very effective for separation of particles which produce TR from those which do not radiate. Hadrons with momenta in the $\mathrm{TeV}$ region emit some amount of TR. Hadron mass difference is relatively small and their identification requires a TRD with multiple TR production thresholds and saturation points (see for instance [14]). In order to produce and detect a number of TR photons sufficient for a good particle separation, the experimental set-up should be a few meters long, thus implying a quite large material budget [15].

An increase of the TR generation threshold and saturation point requires an increase of the foil thickness. That unavoidably leads to the increase of TR photon energies as most of generated radiation occurs with frequencies around $\omega_{\max }=\ell_{1} \omega_{1}^{2} / 2 \pi c$ [3] and, hence, to a decrease of their absorption efficiency particularly in gaseous detectors. A few TRD concepts based on indirect registration of TR photons were proposed for particle separation with high $\gamma$-factors. One of them uses a thin X-ray absorber made of a material with a high yield of fluorescent photons after the TR photo-absorption. Fluorescent photons are registered by the detectors placed outside of the beam area [16]. Another TRD approach is based on the Compton scattering of TR photons in the radiator material $[17,18]$. In this case Compton scattered photons are detected by X-ray sensors placed around the radiator.

One of the possibilities to enhance the PID performance of TRDs is a simultaneous measurement of the number of $X$ rays, their energies and the angles at which they are produced. The major constraint for such detectors is the separation of the absorbed photons from the particle track. This kind of measurement requires a high-granularity detector with high X-ray detection efficiency. Since TR photons are emitted at small angles with respect to the particle, a distance of the order of $1 \mathrm{~m}$ between the radiator and the detector is required to ensure particlephoton separations at the level of a hundred $\mu \mathrm{m}$. A few attempts of using the TR angular information for PID were made in the past with gaseous detectors. It was shown that, indeed, it improves PID capabilities of the detector but the spatial resolution was not enough to reach a good performance $[9,19]$. An alternative approach is based on fine granularity semiconductor detectors. A first attempt to measure the TR angular distribution was made in [20], where a thin DEPFET silicon pixel matrix with $20 \mu \mathrm{m}$ pixel size was used. In order to increase the effective thickness of the detector the particle beam was directed at an angle of $40^{\circ}$ to the detector plane. The experimental results showed a quite good agreement with GEANT4 simulations, however, the radiator-detector geometry did not allow to make detailed studies.

Measurements of the TR photon energies and production angles using a $300 \mu \mathrm{m}$ thick double-sided silicon strip detector (DSSD) with a readout strip pitch of $50 \mu \mathrm{m}$ were performed in [21]. Quite good agreement between data and Monte Carlo (MC) simulations was obtained selecting events with one absorbed photon. However, detectors with the strip readout have limited capabilities to resolve of several detected photons.

The development of high granularity pixellated readout chips/ASICs [22] connected to thick Si or GaAs sensors opens new possibilities to create efficient TR detectors with good separation of the charged particle and TR photons. Preliminary results of TR measurements with a $480 \mu \mathrm{m}$ thick Si-sensor attached to a Timepix 3 chip were presented in [23]. In the present paper the results of detailed studies of the TR photon production in different radiators using such detector are reported. Experimental results are compared with the MC predictions, which include detailed TR and detector response simulations. The measurements were carried out at the CERN SPS facility with a $20 \mathrm{GeV} / \mathrm{c}$ mixed electron and pion beam, and with 120 and $180 \mathrm{GeV} / \mathrm{c}$ muon beams crossing different types of radiators.

\section{Detector description and test beam setup}

\subsection{The TimePix 3 Si detector}

The Medipix and Timepix family of pixel readout chips have been developed at CERN to explore the applicability of energy sensitive pixel detectors outside the field of HEP [24]. However, rich functionalities of these chips also open new possibilities for HEP detectors.

The measurements described in the present paper have been carried out using a $480 \mu \mathrm{m}$ thick p-on-n silicon sensor bonded to a $700 \mu \mathrm{m}$ thick Timepix3 chip [25]. The chip is then attached to the $1.6 \mathrm{~mm}$ thick PCB which provided connections to external services. The chip is composed of two main parts: the pixel matrix and the periphery. The pixel matrix consists of $256 \times 256$ square pixel readout units (pixels) with a $55 \mu \mathrm{m}$ pitch. Each pixel implements an analog front-end with a preamplifier, a leakage current compensation circuit and a discriminator. The discriminator threshold is set both globally and locally. The latter is used for fine tuning of inter-pixel threshold mismatches. Individual pixels can operate in any of the following measurement modes:

- charge (Time over Threshold, ToT) and time (Time of Arrival, ToA)

- time only

- event counting, with integral charge information

Such versatility makes the chip a useful tool for different types of applications. In the test beam studies discussed in this paper, the charge-and-time mode was used. The timing resolution of the pixel stems from the local $640 \mathrm{MHz}$ clock, and is thus $1.56 \mathrm{~ns}$. The ToT measurement provides information about the amount of charge collected by 

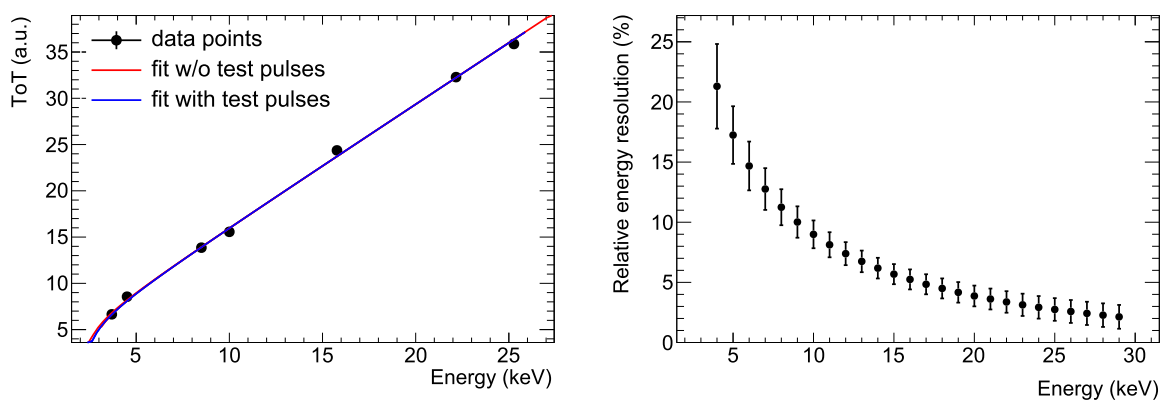

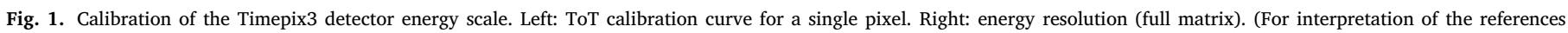
to color in this figure legend, the reader is referred to the web version of this article.)

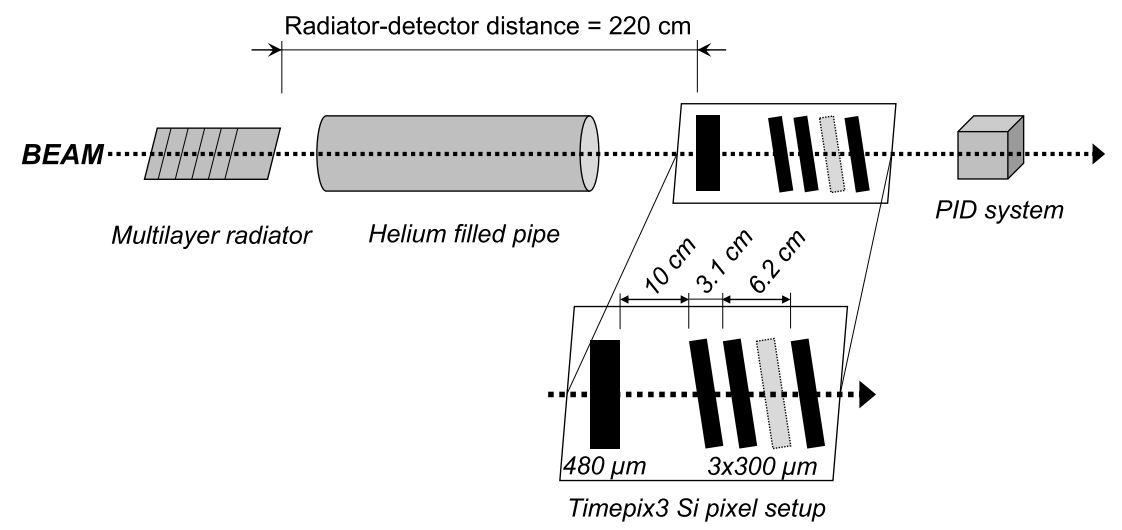

Fig. 2. Scheme of the experimental setup. The beam direction corresponds to Z-axis of the reference frame.

Table 1

List of the available fluorescence targets used for the calibration of the Timepix 3 detector.

\begin{tabular}{llllll}
\hline Target & $\mathrm{Z}$ & $\mathrm{K}_{\alpha 1}[\mathrm{keV}]$ & $\mathrm{K}_{\alpha 2}[\mathrm{keV}]$ & $\mathrm{K}_{\beta 1}[\mathrm{keV}]$ & $\mathrm{K}_{\beta 2}[\mathrm{keV}]$ \\
\hline $\mathrm{Ca}$ & 20 & 3.691 & 3.688 & 4.012 & - \\
$\mathrm{Ti}$ & 22 & 4.510 & 4.504 & 4.931 & - \\
$\mathrm{Cu}$ & 29 & 8.047 & 8.027 & 8.904 & 8.976 \\
$\mathrm{Ga}$ & 31 & 9.251 & 9.234 & 10.263 & 10.365 \\
$\mathrm{As}$ & 33 & 10.543 & 10.507 & 11.725 & 11.863 \\
$\mathrm{Zr}$ & 40 & 15.774 & 15.690 & 17.666 & 17.969 \\
$\mathrm{Ag}$ & 47 & 22.162 & 21.988 & 24.942 & 25.454 \\
$\mathrm{Sn}$ & 50 & 25.270 & 25.042 & 28.483 & 29.106 \\
\hline
\end{tabular}

the pixel. It has a range of 10 bits, with a configurable resolution that can be set via the discharge current of the pre-amplifier. The typical operational conditions give a correspondence of each ToT count to a charge of about $200 \mathrm{e}^{-}$. Here and later $\mathrm{e}^{-}$is the charge of a single electron. Due to the quite small capacitance at the input of the amplifier its noise level is less than $100 \mathrm{e}^{-}$RMS, which corresponds to about $360 \mathrm{eV}$ in the energy scale. The chip operation threshold is chosen to ensure effectively no noise hits while allowing for high detection efficiency for multi-pixel clusters. Usually it is close to $1000 \mathrm{e}^{-}$or $3.6 \mathrm{keV}$ for Si-sensors.

In our studies the data-driven mode of Timepix 3 operation was used: the hit triggers the read-out as soon as the signal at any pixel exceeds the threshold. In data-driven mode the matrix can be readout at a dead time of $475 \mathrm{~ns}$. The sensor was biased at $200 \mathrm{~V}$. In order to eliminate random noise, which could trigger readout during calibration and test beam studies, it was sufficient to set a threshold equivalent to about $3.4 \mathrm{keV}$ for each pixel.

Prior to the measurement, a detailed calibration of the charge scale from ToT units to $\mathrm{keV}$ was performed using X-ray fluorescence on a per-pixel basis. For this purpose the $\mathrm{K}_{\alpha}$ emission photons from the targets listed in Table 1 were used.
An example of a single pixel calibration curve is shown on the left plot of Fig. 1. In the low energy range, where the linearity is lost, the calibration curve was mapped using the charge injection functionality of Timepix3. The calibration data points were fitted by a function of the form [26]:

$\mathrm{ToT}=a E+b-\frac{c}{E-E_{0}}$,

where $E$ is the photon energy and $E_{0}$ is the constant energy term related to a shift of the energy scale. The energy resolution of the detector as a function of photon energy is shown in the right plot of Fig. 1. The measured cluster energy resolution approximately follows the formula:

$\sigma(E) / E=0.94 \times E^{-1.04}$,

where $E$ is measured in $\mathrm{keV}$.

\subsection{Test beam setup}

The detector was exposed to the particle beam at the CERN SPS H8 beam line. The beams used in these studies were a $20 \mathrm{GeV} / \mathrm{c}$ mixed electron-pion beam and muon beams with momenta of $180 \mathrm{GeV} / \mathrm{c}$ and $120 \mathrm{GeV} / \mathrm{c}$ (this corresponds, for electrons $\gamma \approx 3.9 \times 10^{4}$ and for muons $\gamma \approx 1.7 \times 10^{3}$ and $\gamma \approx 1.13 \times 10^{3}$, respectively). A schematic diagram of the test beam setup is shown in Fig. 2 .

Particle identification was implemented at the trigger level. The trigger system included signals from scintillator counters, a Cherenkov counter, a pre-shower detector and a lead glass calorimeter. A multiplicity counter was also used to veto upstream showers and multiparticle events. High purity trigger signals corresponding to the particle species were sent to the Timepix3 setup as trigger flags. Contamination of particles of the other sorts for each trigger type was below $10^{-4}$.

In order to increase the separation between the X-rays and the parent beam particle, the TR radiators were placed at a distance of $220 \mathrm{~cm}$ from the tested silicon detector. In between, a helium-filled 


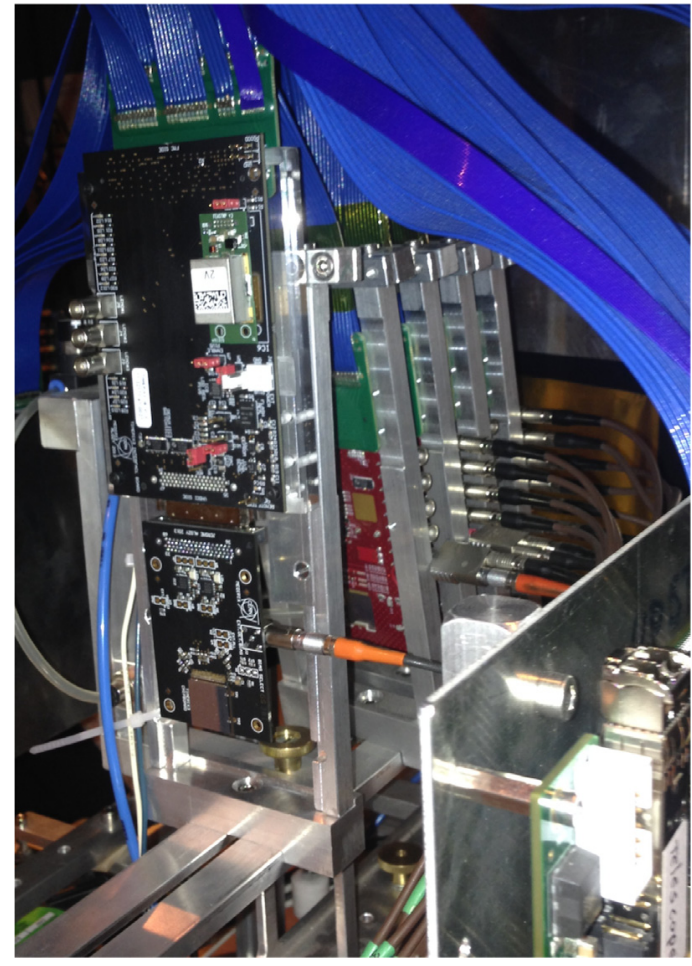

Fig. 3. The Timepix3 telescope.

Table 2

Parameters of radiators used in the beam test: $l_{1}$ and $l_{2}$ are the thickness of the foils and of the size of the gaps, respectively, $N_{f}$ is the number of foils.

\begin{tabular}{lllll}
\hline Radiator & Foil/gap material & $l_{1}$ & $l_{2}$ & $N_{f}$ \\
\hline Mylar (1 set) & Mylar/air & $50 \mu \mathrm{m}$ & $2.97 \mathrm{~mm}$ & 30 \\
Mylar (3 sets) & Mylar/air & $50 \mu \mathrm{m}$ & $2.97 \mathrm{~mm}$ & 90 \\
Polyethylene (1 set) & Polyethylene/air & $270 \mu \mathrm{m}$ & $3.3 \mathrm{~mm}$ & 30 \\
Polyethylene (3 sets) & Polyethylene/air & $270 \mu \mathrm{m}$ & $3.3 \mathrm{~mm}$ & 90 \\
Polypropylene (5 sets) & Polypropylene/air & $15 \mu \mathrm{m}$ & $222 \mu \mathrm{m}$ & 180 \\
Dummy radiator & Polyethylene & $3 \mu \mathrm{mm}$ & & 1 \\
\hline
\end{tabular}

pipe with windows made from $\sim 16 \mu$ m polyethylene foils was placed to minimize the TR photon absorption.

Different types of radiators were used in the tests (see Table 2). Radiators were made as sets, and each set was assembled inside its own support frame. The radiators consisting of multiple sets are stacked in such a way that the distance between foils of adjacent sets is the same as within one set (with the exception of the polypropylene radiator where the distance between radiator sets was about $4.5 \mathrm{~mm}$ ).

The tested Timepix 3 detector was implemented as a part of the LHCb VELO test beam telescope $[27,28]$. The LHCb VELO Timepix3 telescope is a silicon pixel tracking system initially built to evaluate the performance of LHCb VELO Upgrade prototypes. This detector consists of 8 modules of $300 \mu \mathrm{m}$ thick silicon sensors, bump-bonded to Timepix 3 ASICs with the same pixel size as the tested detector. For our studies we used only one arm of this setup, which reads out 4 modules. The tested silicon detector, positioned perpendicular to the beam, was the first plane along the beam line. Three downstream planes of the telescope, shown in Fig. 2 in black, were used for tracking purposes. To improve the spatial resolution by maximizing charge sharing between the pixels, the three tracking planes were tilted by $9^{\circ}$ about the X- and Y- axes with the $Y$-axis being vertical (see Fig. 3). The distance between the planes of the telescope along the $Z$-axis is $31 \mathrm{~mm}$ and the distance from the tested sensor to the first tracking plane is $100 \mathrm{~mm}$. The integration of the four detectors into the SPIDR readout ensured that the data from all planes were synchronized [29]. In addition, the readout was configured to accept two trigger inputs from the PID system, one corresponding to particles identified as electrons, the other to pions (or muons). Such flags were added to the output data so that the particle identification information is available for offline analysis.

\section{Data analysis}

\subsection{Event reconstruction}

The Timepix3 telescope is operated in self trigger mode and provides a continuous data stream. Similarly, the PID system delivers triggers when a particle is detected. Both systems share a global time reference, and all pixel hits and trigger signals have time stamps which are used to synchronize the two data streams offline. For each trigger signal an event is created. The trigger from the PID system arrives later than signals registered in the Timepix 3 chip by about $\Delta t=t_{\text {trigger }}-$ $t_{\text {pixel }}=400 \mathrm{~ns}$. The pixel hits on all Timepix3 chips with the time stamp within a window of $200 \mathrm{~ns}$ around this time are assigned to the event. Reconstructed events are stored as ROOT trees. Before any further analysis, the outermost 5 columns and rows of the pixel matrix are removed from the data. This is done due to the fact that pixels at the edge of the matrix tend to be noisier. Additionally, any other noisy pixels are removed by using the noise scan procedure of the Proteus software package for test beam analysis [30]. For all data taken, the fraction of masked pixels within a defined active area is well below $0.05 \%$ on all chips.

\subsection{Clustering method}

A cluster is defined as a group of adjacent hit pixels surrounded by empty pixels. The $i$ th and $j$ th pixels are considered adjacent if their positions are such that

$$
(\Delta X=1 \wedge \Delta Y=0) \|(\Delta X=0 \wedge \Delta Y=1),
$$

where $\Delta X=\left|X_{i}-X_{j}\right|$ and $\Delta Y=\left|Y_{i}-Y_{j}\right|$ are the differences between their $X$ and $Y$ coordinates in pixel units. Once all pixels are grouped in clusters, the cluster energies are calculated by summing up the energies of all pixels in the cluster. Since X-rays are mainly absorbed in the front sensor and materials behind it, in the downstream silicon planes events contain only a single cluster, produced by the charged particles. In the first silicon sensor we expect events with multiple clusters, produced by the charged particles and by the eventual TR photons. In fact, if a TR photon is absorbed at sufficient distance from the charged particle it will produce a cluster of pixels separated from the one produced by the particle. In the left plot of Fig. 4 the cluster energies against the cluster sizes (i.e. the number of pixels in a cluster) are plotted for a run with $20 \mathrm{GeV} / \mathrm{c}$ electrons crossing the 90 -foil Mylar radiator. One sees that there are two main families of clusters: large size clusters with energies above $80 \mathrm{keV}$ correspond to particles, while small size clusters with lower energies correspond to TR photons. The typical cluster size is 5-6 for particles and 1-2 for photons. In the right plot of Fig. 4 the distribution of the number of TR clusters per charged particle is shown. This distribution follows the Poisson law with the average number of detected photons of about 1 .

The left plot in Fig. 5 shows the energy distributions of all clusters detected by the front sensor (red histogram) at the same experimental conditions. The energy deposition of charged particles in the Si-sensor is significantly larger than that of the TR photons and it can be identified as the most energetic cluster in the event. The energy distribution of most energetic clusters in the event is shown in the same plot (blue histogram). Since the aperture of the trigger counters is larger than the detector size, a few particles can miss the sensitive area of the chip. The small peak below $20 \mathrm{keV}$ in the most energetic cluster distribution corresponds to such events, where only TR photons are detected. For a comparison, in the right plot of Fig. 5 the energy distribution of the most energetic clusters for a $180 \mathrm{GeV} / \mathrm{c}$ muon run without radiator is shown. The cluster energy is a very clear separation parameter. In the analysis only events with a maximum energy cluster above $80 \mathrm{keV}$ were selected to ensure the presence of the particle. 

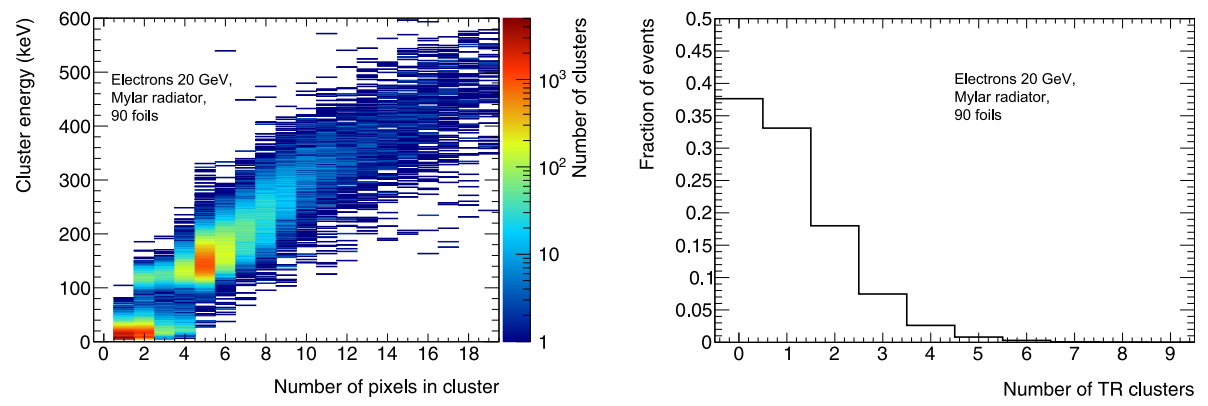

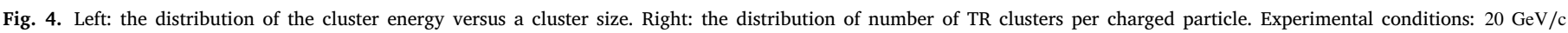
electrons, 90-foil Mylar radiator.
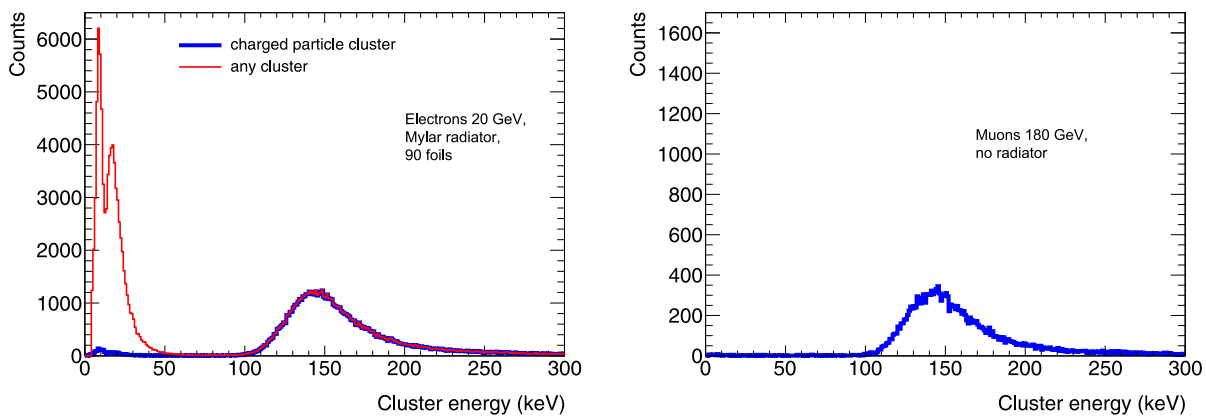

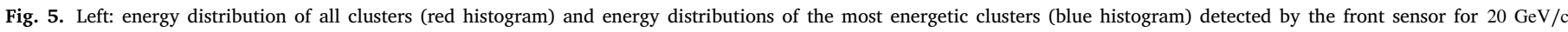

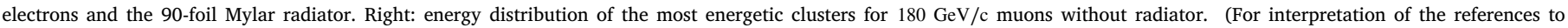
color in this figure legend, the reader is referred to the web version of this article.)

\subsection{Particle cluster position accuracy}

The accuracy of the particle position measurement with the front $480 \mu \mathrm{m}$ sensor was studied using the tracks reconstructed by the 3plane telescope. Tracking and alignment procedures are similar to those described in details in [31]. The position of a cluster in local plane coordinates is assigned by calculating the energy-weighted average of the individual pixel positions, i.e.:

$x_{C O G}=\frac{\sum_{j=1}^{n} \omega_{j} x_{j}}{\sum_{j=1}^{n} \omega_{j}}$
$y_{C O G}=\frac{\sum_{j=1}^{n} \omega_{j} y_{j}}{\sum_{j=1}^{n} \omega_{j}}$,

where $n$ is the number of pixels in the cluster, $\omega_{j}$ is the energy of the $j$ th pixel in the cluster and $\left(x_{j}, y_{j}\right)$ are its coordinates. The suffix "COG" stands for "center of gravity".

To be selected for further analysis, it is required that each of the 3 telescope planes has only one cluster with an energy above $60 \mathrm{keV}$ in a given event. A straight line fit is performed using these clusters and the resulting track is then projected backwards onto the front sensor. Clusters of the front sensor are not used for the fit as this would bias the resulting tracks. Examples of the distributions of the track-cluster residuals (Y-axis) for one of the telescope planes (left) and for the front sensor (right) are shown in Fig. 6. The intrinsic accuracy of the telescope planes measured using 8 detector planes [28] is about $5 \mu \mathrm{m}$. The average telescope plane accuracy obtained in our measurements is about $8 \mu \mathrm{m}$. The difference is explained by the fact that the residual distribution is defined by the intrinsic accuracy of each detector plane, by their alignment precision and by the track reconstruction accuracy. Last two components are larger in our case because only 3 detector planes are used for the track reconstruction while in [28] 8 detector planes were used. On the front sensor, the measured track-cluster residual distribution has a sigma of $15.7 \mu \mathrm{m}$ (right plot of Fig. 6). The track position prediction accuracy on the front sensor is affected by the telescope plane accuracy. For the measured values the simulated extrapolation accuracy of the particle position for the front sensor is about $16.4 \mu \mathrm{m}$. Which is already above the obtained value of $15.7 \mu \mathrm{m}$. If, instead, we use the intrinsic accuracies of the telescope planes $(5 \mu \mathrm{m})$, the estimated track prediction accuracy would be about $11 \mu \mathrm{m}$. The latter gives the upper limit for the intrinsic accuracy of the $480 \mu \mathrm{m}$ sensor of about $11.2 \mu \mathrm{m}$. One concludes that the cluster position measurement accuracy for particles in the front sensor is much better than that for photons which is largely defined by the pixel size and in the following analysis only information from the front sensor was used.

\subsection{Photon cluster reconstruction efficiency}

A close-up view of the distribution of the cluster coordinates for the absorbed TR photons around the beam particle $(20 \mathrm{GeV} / \mathrm{c}$ electrons and the 30 foil Mylar radiator) is shown in the left plot of Fig. $7 .{ }^{1}$ The position of the beam particle cluster is always placed at the coordinate origin. The four empty pixels in the center of the plot correspond to the area where TR photon clusters cannot be reconstructed since they will overlap and merge with the particle cluster. The TR photon cluster reconstruction efficiencies as a function of the distance from the beam particle cluster for data and MC are shown in the right plot of Fig. 7. These efficiencies are calculated as the fraction of analyzed events for which a pixel at a certain distance from the beam particle cluster position belongs to a TR photon cluster. After a distance equivalent to 3 times the pixel pitch, a $100 \%$ efficiency of the TR cluster reconstruction is reached. At this distance the beam particle cluster and the TR photon cluster will always be separated. A separation of the TR photon cluster from the beam particle cluster at smaller distances is also possible, but depends on the shape of both clusters. A distance of 3 pixels corresponds to $165 \mu \mathrm{m}$ and makes it possible to detect TR photons at angles down to $0.075 \mathrm{mrad}$ if the TR photon is emitted $2 \mathrm{~m}$ before the

${ }^{1}$ On this and following two-dimensional plots entries are normalized to the total number of particles. 

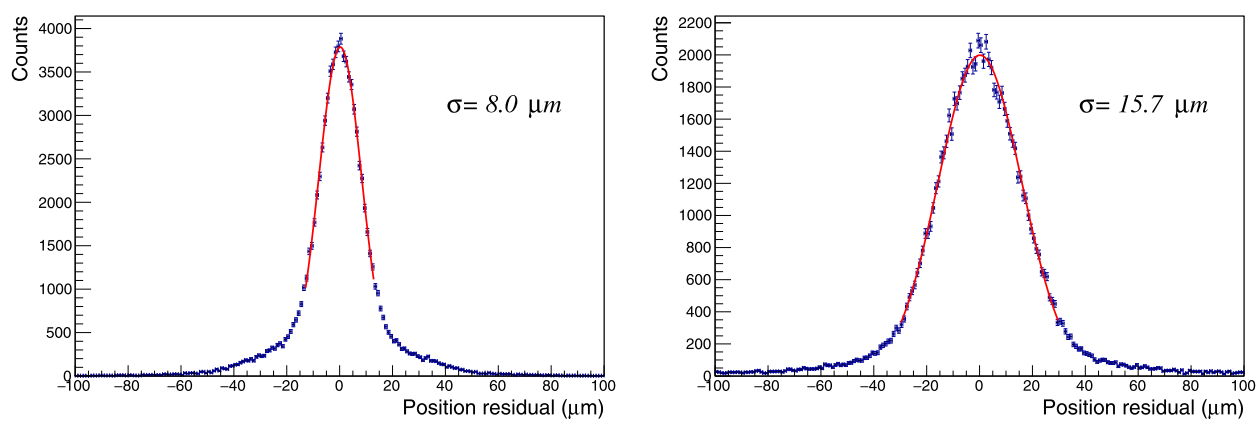

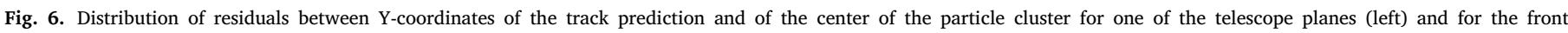
detector (right). The distributions were fitted with a Gaussian within the range $[-20,20] \mu \mathrm{m}$ to avoid fitting tails likely caused by TR photons.


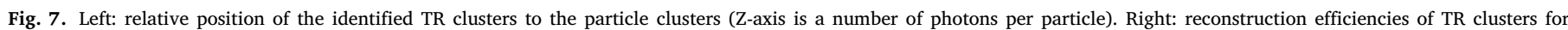

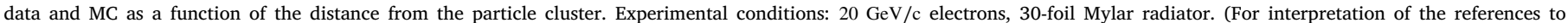
color in this figure legend, the reader is referred to the web version of this article.)

detector. Notice that although the bins for both plots in Fig. 7 have the same dimension as pixels on the Timepix 3 chip $(55 \mu \mathrm{m})$, they do not correspond to physical pixels because the coordinate origin is always taken at the COG of the particle cluster.

\section{Simulation model}

The Monte Carlo (MC) model used for an interpretation of the experimental data includes simulation of the physics processes and of the detector response. The format of the simulation output is the same as that of data after calibration and event selection. The procedures described in the previous section were used for reconstruction of both the simulated events and the real data events.

\subsection{Transition radiation simulation model}

When the ionizing particle travels through the radiator elements, it can emit TR X-rays. Radiators used in these studies had different production qualities. The Mylar radiator was made with a quite high precision, and its parameters have variations of a few percents. The polypropylene radiator has a small variation of the material thickness but a significant variation of the space between foils. For the polyethylene radiator both parameters significantly fluctuate. In order to describe the observed results two simulation models were used.

\subsubsection{Regular radiator}

The first approach assumes the regular structure of radiators. In that case a differential two-dimensional energy-angle distribution of the TR $\mathrm{X}$-rays is described by [32]:

$\frac{d^{2} N_{\text {gen }}}{d \theta d \omega}=4 \frac{d^{2} N_{0}}{d \theta d \omega} \sin ^{2}\left(\frac{l_{1}}{Z\left(\omega_{1}\right)}\right) \frac{\sin ^{2}\left(N\left(\frac{l_{1}}{Z\left(\omega_{1}\right)}+\frac{l_{2}}{Z\left(\omega_{2}\right)}\right)\right)}{\sin ^{2}\left(\frac{l_{1}}{Z\left(\omega_{1}\right)}+\frac{l_{2}}{Z\left(\omega_{2}\right)}\right)}$, where

$\frac{d^{2} N_{0}}{d \theta d \omega}=\frac{1}{c}\left(\frac{q e}{4 \pi c}\right)^{2} \theta^{3} \omega\left(Z\left(\omega_{1}\right)-Z\left(\omega_{2}\right)\right)^{2}$

and

$$
Z\left(\omega_{i}\right)=Z\left(\theta, \omega, \omega_{i}\right)=\frac{4 c}{\omega\left(\gamma^{-2}+\left(\frac{\omega_{i}}{\omega}\right)^{2}+\theta^{2}\right)} .
$$

In these formulas $N_{\text {gen }}$ is the number of generated TR photons, $\omega$ is the X-ray energy, $\theta$ is the emission angle of X-rays with respect to the radiating particle, $\omega_{1}$ and $\omega_{2}$ are the plasma frequencies of the foil and gap materials, $l_{1}$ and $l_{2}$ are the thickness of the foils and the distance between them, $N$ is the number of foils in the radiator block, $Z\left(\omega_{1}\right)$ and $Z\left(\omega_{2}\right)$ are the formation zones in the foil and gap materials, $\gamma$ is the Lorentz factor of the radiating particle. The right-hand side of Eq. (3) includes 3 factors. The first two describe the TR spectra of a single foil, while the last factor corresponds the TR interference from $N$ foils.

\subsubsection{Irregular radiator}

If the radiator has significant fluctuations of the parameters, the regular radiator approach cannot be used. For simulations of irregular radiators, the Garibian formulas were used instead [33]. In this approach it is assumed that $l_{1}$ and $l_{2}$ follow gamma distributions. Formulas describing the TR distribution for irregular radiators are rather complex and not presented here. This approach was used to obtain TR distribution predictions for the polypropylene and the polyethylene radiators. In the case of very small parameter variations they reduce to the distribution of Eq. (3). Calculations show that fluctuations of $l_{1}$ and $l_{2}$ do not significantly affect the total TR X-ray yield, but lead to deformation of the energy spectrum and to smearing of the angular distribution. 


\subsubsection{Photon propagation in the radiator}

The average number $N_{\gamma}$ of TR photons produced by a particle with Lorentz factor $\gamma$ crossing a given radiator is calculated by integrating Eq. (3) in the case of regular radiators or the corresponding doubledifferential spectrum obtained from Garibian's formulas in the case of irregular radiators. Only photons produced in the ranges $4 \mathrm{keV} \leq$ $\omega \leq 60 \mathrm{keV}$ and $0 \leq \theta \leq 4 \mathrm{mrad}$ were considered in the simulations. The low energy limit is defined by a strong absorption of soft photons in the materials on the way to the detector and also by the detector sensitivity range. The upper energy limit is defined by the ability of the Si-sensor to absorb high energy photons. The number of TR photons produced in each event is then extracted from a Poisson distribution with a mean value $N_{\gamma}$. It is assumed that photons can be emitted at any foil inside the radiator with equal probabilities. The energy and the emission angle of each photon are randomly extracted from the doubledifferential TR spectrum. The trajectories and the potential interception points of the photons with the detector are then determined. Photons with their interception points lying outside the detector active area are disregarded.

\subsubsection{Absorption of TR X-rays}

The absorption of the X-rays is taken into account in all the radiator and detector materials: radiator foils, air, thin polyethylene windows of the helium pipe, helium itself, the aluminum coating of the sensor and the sensor material (silicon). The absorption probability in the $i$ th absorber (air, $\mathrm{Si}, \mathrm{He}, \ldots$ ) is given by $p_{i}(\omega)=1-e^{-L_{i} / \lambda_{i}(\omega)}$, where $\omega$ is the photon energy, $L_{i}$ is the length of the absorber and $\lambda_{i}(\omega)$ is its absorption length. All absorption processes (coherent and non-coherent scattering, photoelectric effect) are taken into account. The values of the absorption lengths were calculated using the mass absorption coefficients taken from the NIST reference database [34].

\subsubsection{Multiple scattering effects}

In the experiment the TR emission angle is calculated on the basis of the coordinate difference between the particle and the photon clusters. Multiple scattering of the particle in the radiator material after the TR production point leads to a deviation of the particle cluster position from that which would correspond to the particle trajectory without scattering. This results in a smearing of the photon angular distribution. This effect is not significant for high momentum particles (muons above $100 \mathrm{GeV} / \mathrm{c}$ ), but for the $20 \mathrm{GeV} / \mathrm{c}$ electron beam and for large radiator thickness it cannot be neglected. ${ }^{2}$

The simulation of the impact of multiple scattering on the photon angle measurements requires detailed knowledge of the particle position and its direction in each TR production point. In this work a simplified method was used. The space angle of the scattered particle is extracted from a two dimensional Gaussian distribution with a sigma given by the expression [4]:

$\sigma_{\theta}=\sqrt{2} \frac{13.6 \mathrm{MeV}}{\beta c p} \sqrt{\frac{x}{X_{0}}}\left[1+0.038 \ln \left(\frac{x}{X_{0}}\right)\right]$,

where $x$ is the thickness of the radiator crossed by the particle after TR production and $X_{0}$ is the radiation length of the material. The impact point of the particle on the detector plane is obtained using the space angle and the distance $L$ between the radiator and the detector, and assuming a polar angle symmetry of the scattering process with respect to the beam direction (Z-axis). The coordinate differences between the particle impact point and its position without scattering are then added to the photon impact point coordinates. The same procedure is repeated for each TR photon if more than one photon is produced by the same particle. In this approach the final position of the particle cluster is the same as in the case without scattering, while the positions of the photon clusters are perturbed.

2 The multiple scattering angle for $20 \mathrm{GeV} / \mathrm{c}$ electrons crossing the 90 foils mylar radiator (total thickness $x / X_{0} \approx 0.016$ ) is $\theta_{0} \approx 0.11 \mathrm{mrad}$.

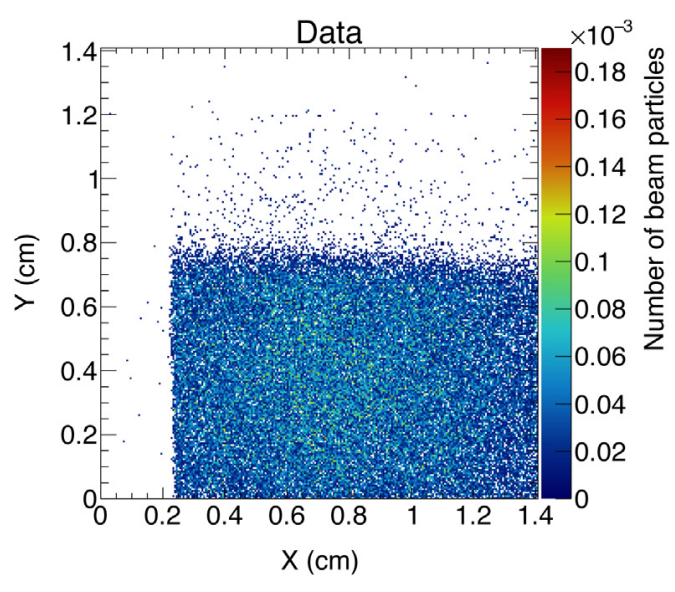

Fig. 8. Distribution of the beam particle impact point for data. $Z$-axis is normalized on the total mumber of events. The data have been collected with a $20 \mathrm{GeV} / \mathrm{c}$ electron beam crossing the 90 foils Mylar radiator.

\subsection{Detector simulation model}

\subsubsection{Ionizing particles}

During the beam tests the particle beam profile varies with the operation conditions. In addition, the trigger counter was misplaced with respect to the Si sensor. In order to take these effects into account in the MC simulations we used the distributions of the impact points of particles taken directly from the data of each run. Fig. 8 shows an example of such distribution.

The energy deposition of the particles in the detector was simulated using the experimental distribution of the energies of particle clusters above $80 \mathrm{keV}$ (see Fig. 5). We assume that the energy loss of the particle is uniform along its track in the silicon active volume. The track then is divided into 10 segments and in each of these segments a point-like cluster of electron-hole pairs is produced. Holes and the electrons drift respectively towards the pixels (cathodes) and towards the back plane (anode) of the sensor. Signals on individual pixels are defined by the collected charge at the end of the drift process. Diffusion processes (will be described later) spread the charge among pixels and the fractions of the charge collected by the various pixels depends on its initial position inside the detector. The charges on each pixel from all track segments are finally combined.

\subsubsection{Photon absorption in the detector}

To simulate photon absorption in the detector material all the processes are taken into account. If a photon is absorbed by the photoelectric effect, all energy is deposited in the interaction point. In case of coherent scattering, no energy is deposited in the interaction point and the photon scattering angle is simulated on the basis of the well-known expression for a probability density function (PDF) for this process:

$f(\theta)=\frac{3}{8}\left(1+\cos ^{2} \theta\right)$.

A similar procedure is applied in case of Compton scattering. The scattering angle is extracted from a PDF derived from the Compton cross section and the residual photon energy $E_{\gamma}^{*}$ after interaction is given by:

$E_{\gamma}^{*}=\frac{E_{\gamma}}{1+\frac{E_{\gamma}}{m_{e} c^{2}}(1-\cos \theta)}$,

where $E_{\gamma}$ is the initial photon energy and $m_{e}$ is the electron mass. The absorption of scattered photons is calculated using new trajectories in the detector sensitive area. When a photon is absorbed the charge corresponding to its energy starts to drift towards the electrodes. 

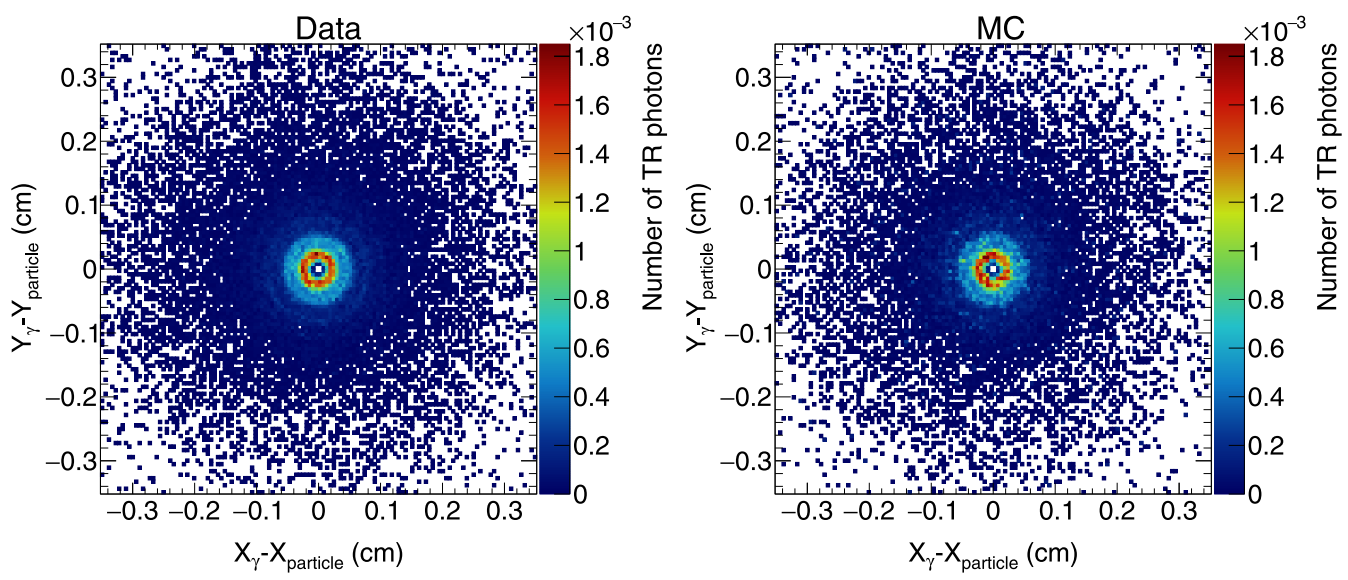

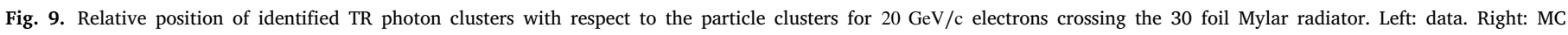
simulation. $Z$-axis is a number of photons per particle.
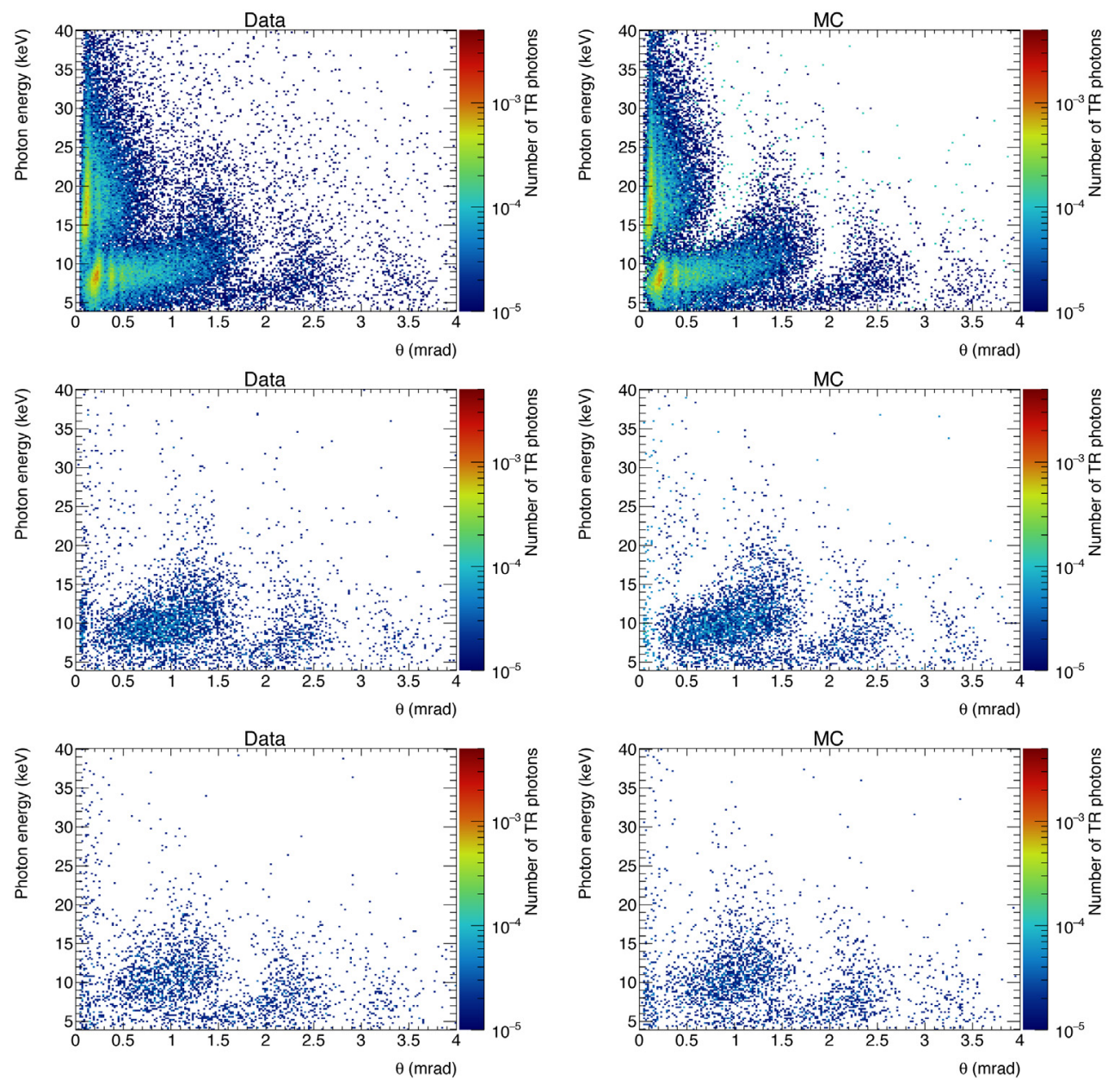

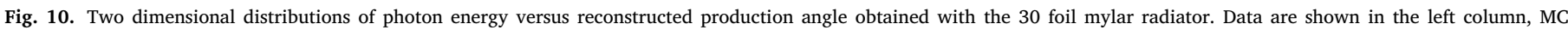

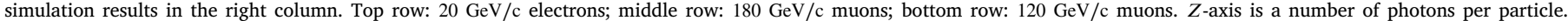

\subsubsection{Detector model parameters}

The diffusion process of drifting charge carriers in the silicon sensor is a complex function of many parameters. The electric field is nonuniform due to the polarization effects of the sensor material. Its distortions in proximity of inter-pixels gaps and other non-uniformities have significant impact on the charge cloud spread. The Coulomb repulsion among carriers must also be taken into account. In simulations, a simplified data-driven model of the charge spread was used [35]. After the charge is collected, the charge density on the pixel plane is considered to have a 2D Gaussian shape with a sigma which linearly 

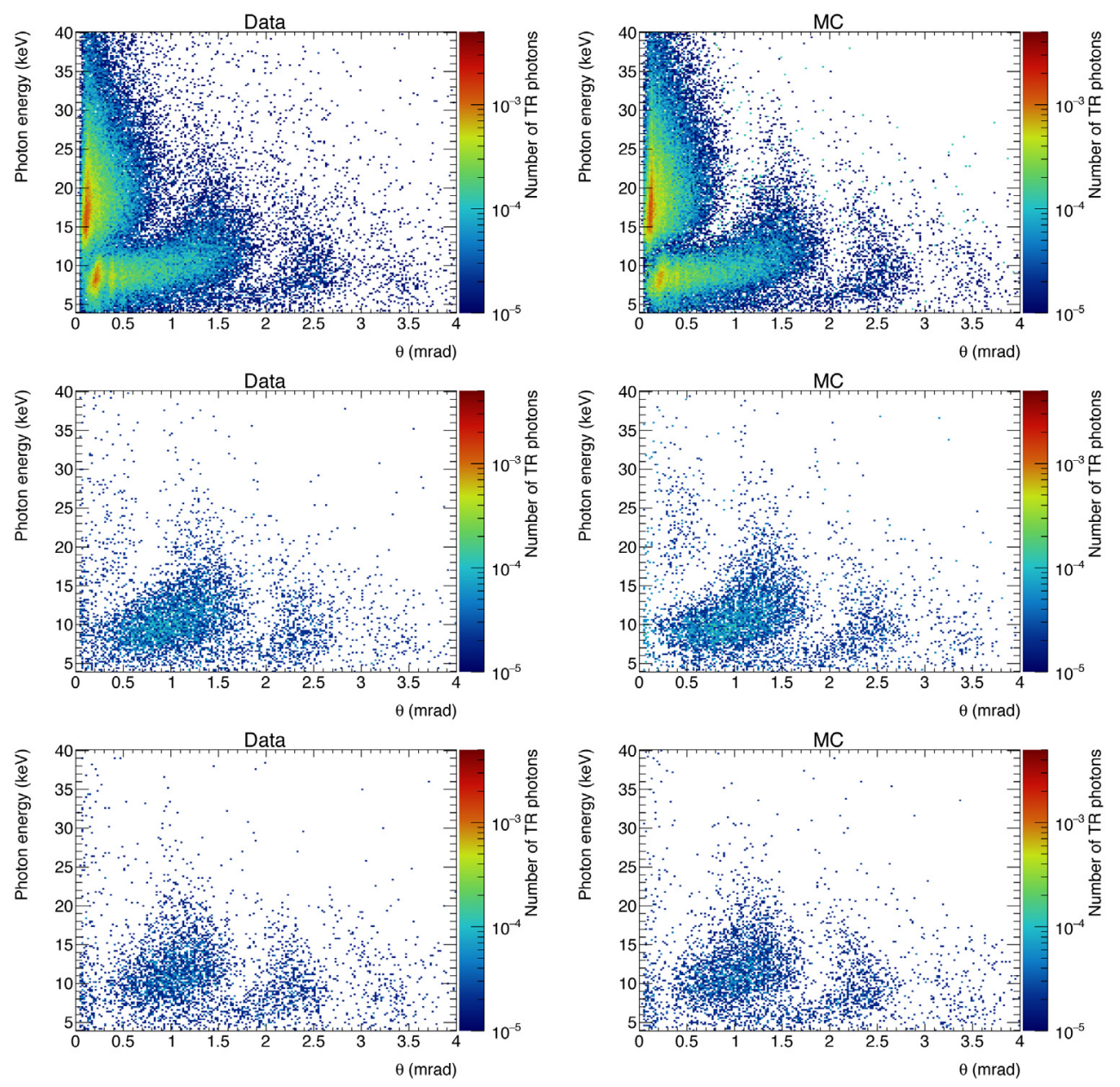

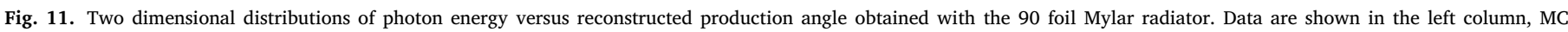

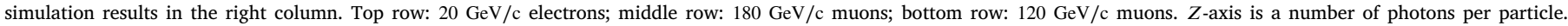

depends on the drift distance:

$\sigma_{d i f f}=a_{d i f f}\left(h_{c h i p}-h_{a b s}\right)+b$,

where $h_{\text {chip }}=480 \mu \mathrm{m}$ is the silicon chip thickness and $h_{a b s}$ is the depth where the charge carriers are created. Parameters $a_{\text {diff }}$ and $b$ are then defined on the basis of the best fit to the data.

After the charge is collected by each pixel and its corresponding energy is defined, the noise is added. The noise level of each pixel expressed in energy units is taken equal to $400 \mathrm{eV}$. This value is a bit larger than the noise of the electronics because it includes the threshold uncertainties and the ToT binning. The energy associated with each pixel is then smeared using the Gaussian distribution with a $\sigma$ of $400 \mathrm{eV}$. The averaged electronics threshold used in the test-beam studies was equivalent to $3.4 \mathrm{keV}$. The fraction of charge collected by a pixel will be lost if it is below the pixel electronics threshold. Since the electronics calibration is not very precise at low signal levels, the electronics threshold in simulations has been taken as a tuning parameter.

The simulation model was tuned using $20 \mathrm{GeV} / \mathrm{c}$ electron runs with the 30 foils Mylar radiator, whose parameters are measured with high accuracy and which exhibits negligible fluctuations in the thicknesses of the foils and of the air gaps. The soft part of the energy spectrum is quite sensitive to the charge diffusion and the electronics threshold. Deviations from correct values lead to the displacement of the low energy TR peak position and to the deformation of its shape. It was found that the best parameters which define the sigma of the charge distribution are $a_{\text {diff }}=0.004$ and $b=1 \mu \mathrm{m}$. The optimum electronics threshold is found to be $3.2 \mathrm{keV}$ which is pretty close to the experimental value.
These parameters were used in simulations for all radiators and for all kinds of particles.

\subsubsection{Effects of bremsstrahlung and delta electrons}

In order to take into account secondary processes leading to a production of photons and delta electrons along the beam line, a few runs with a "dummy" radiator were taken. In these runs the radiators were replaced by a $3 \mathrm{~mm}$ thick polyethylene slab. Photon clusters obtained in these runs were added to those obtained in the simulations, with a scaling factor accounting for the different numbers of events.

\section{Simulation and data comparison}

\subsection{Mylar radiators}

Since parameters of the Mylar radiator have minimal fluctuations, in the simulation the Mylar radiators were treated as perfectly regular radiators. Fig. 9 shows the distribution of the relative positions of the absorbed TR photons with respect to the beam particles for $20 \mathrm{GeV} / \mathrm{c}$ electrons in the case of the 30 foil Mylar radiator for the data and MC simulations. The plots show a good qualitative agreement between data and simulation results. TR photons are spread over a rather large area, but most of them are irradiated close to the particle direction and correspond to the first interference maximum.

The results obtained with the 30 foils and 90 foils Mylar radiators are summarized in Figs. 10 and 11, where the measured doubledifferential TR photon spectra as a function of emission angle and 

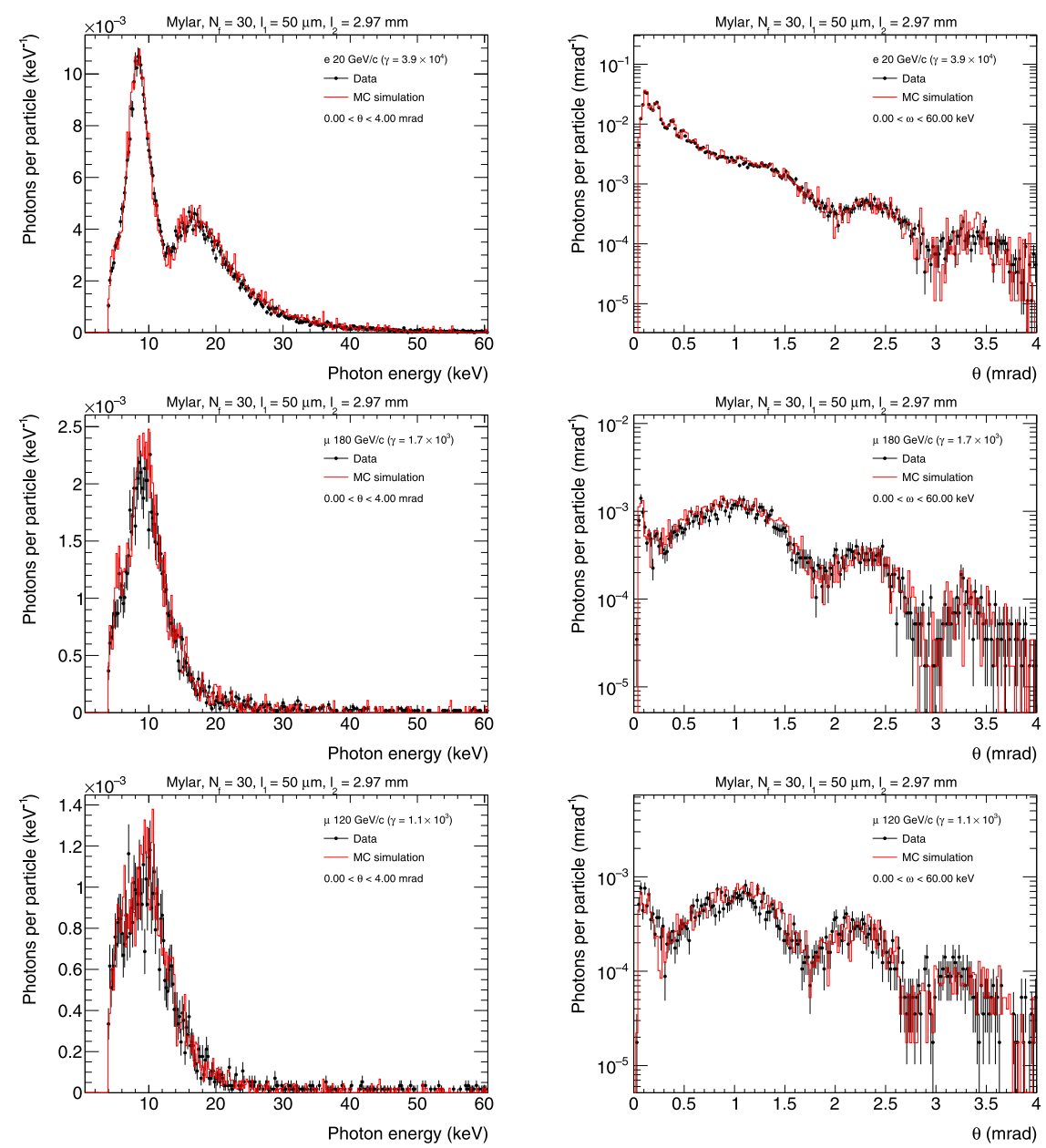

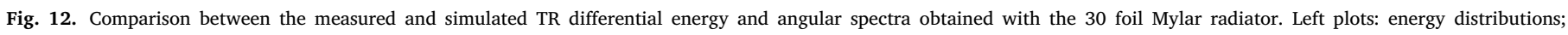

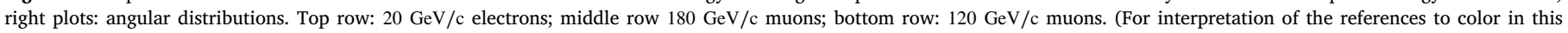
figure legend, the reader is referred to the web version of this article.)

photon energy are compared with those obtained from the MC simulation. The left plots refer to experimental data, while the right plots refer to the MC simulation. The plots for the three beam configurations, with $20 \mathrm{GeV} / \mathrm{c}$ electrons, $180 \mathrm{GeV} / \mathrm{c}$ muons and $120 \mathrm{GeV} / \mathrm{c}$ muons are shown respectively in the top, middle and bottom row of the figures.

From these plots one concludes that, for the Mylar radiator, there are two main families of TR photons. The first one corresponds to photons with energies $\omega>12 \mathrm{keV}$ emitted at angles $\theta<0.5 \mathrm{mrad}$, while the second one corresponds to photons in the remaining region of the $(\theta, \omega)$ plane. The yield of TR photons of the first family has a very strong dependence on the Lorentz factor: it is maximal for $20 \mathrm{GeV} / \mathrm{c}$ electrons $\left(\gamma \sim 3.9 \times 10^{4}\right)$ and practically disappears for $180 \mathrm{GeV} / \mathrm{c}$ muons $\left(\gamma \sim 1.7 \times 10^{3}\right)$. On the other hand, the yield of TR photons of the second family seems to exhibit a mild dependence on the Lorentz factor, even in the high energy region. These features could be exploited to sharpen the dependence of the TRD response on the gamma factor, thus improving its PID performance. Details of the energy and the angular distributions of the TR photons for Mylar radiators with 30 and 90 foils and a comparison with the MC simulations are presented in Figs. 12 and 13. The left plots show the energy distributions, while the right plots show angular distributions. The plots referring to the beam configurations with $20 \mathrm{GeV} / \mathrm{c}$ electrons, $180 \mathrm{GeV} / \mathrm{c}$ muons and $120 \mathrm{GeV} / \mathrm{c}$ muons are shown respectively in the top, middle and bottom rows of the figures.

These figures show that simulations reproduce the experimental data very well, even in details, for all types of particles. No special parameter tuning except what was described in the sections above were done. The TR photon production at large angles weakly depends on $\gamma$. For the 90 foils radiator the multiple scattering significantly smears the interference peaks and this effect is also very well reproduced by the MC simulation.

\subsection{Polypropylene radiator}

Simulations of the transition radiation production for the polypropylene radiator were done using the irregular radiator approach. The polypropylene radiator consists of $15.5 \mu \mathrm{m}$ thick foils spaced by $222 \mu \mathrm{m}$ air gaps (see Table 2). The foil thickness was measured with a high accuracy both mechanically and using a X-ray attenuation technique. It was found that the foils have the same thickness within percent level accuracy (a 1\% fluctuation was taken in the simulation). However, the distance between foils is known as an average value and the radiator production technique cannot guarantee its uniformity with an accuracy better than $10 \%$. This value is difficult to measure, and it was considered in simulations as a tuning parameter. Fluctuations of the spacing between foils do not change the TR energy spectrum but cause a smearing of the peaks in the angular distributions. It was found that the best agreement in angular distributions is reached with the fluctuation of the air gap between foils around 13\%. Another factor which affects TR angular distributions is the distance between blocks of the radiator. The polypropylene radiator used for the test beam studies consists of 5 blocks spaced by about $4.5 \mathrm{~mm}$. This means that 

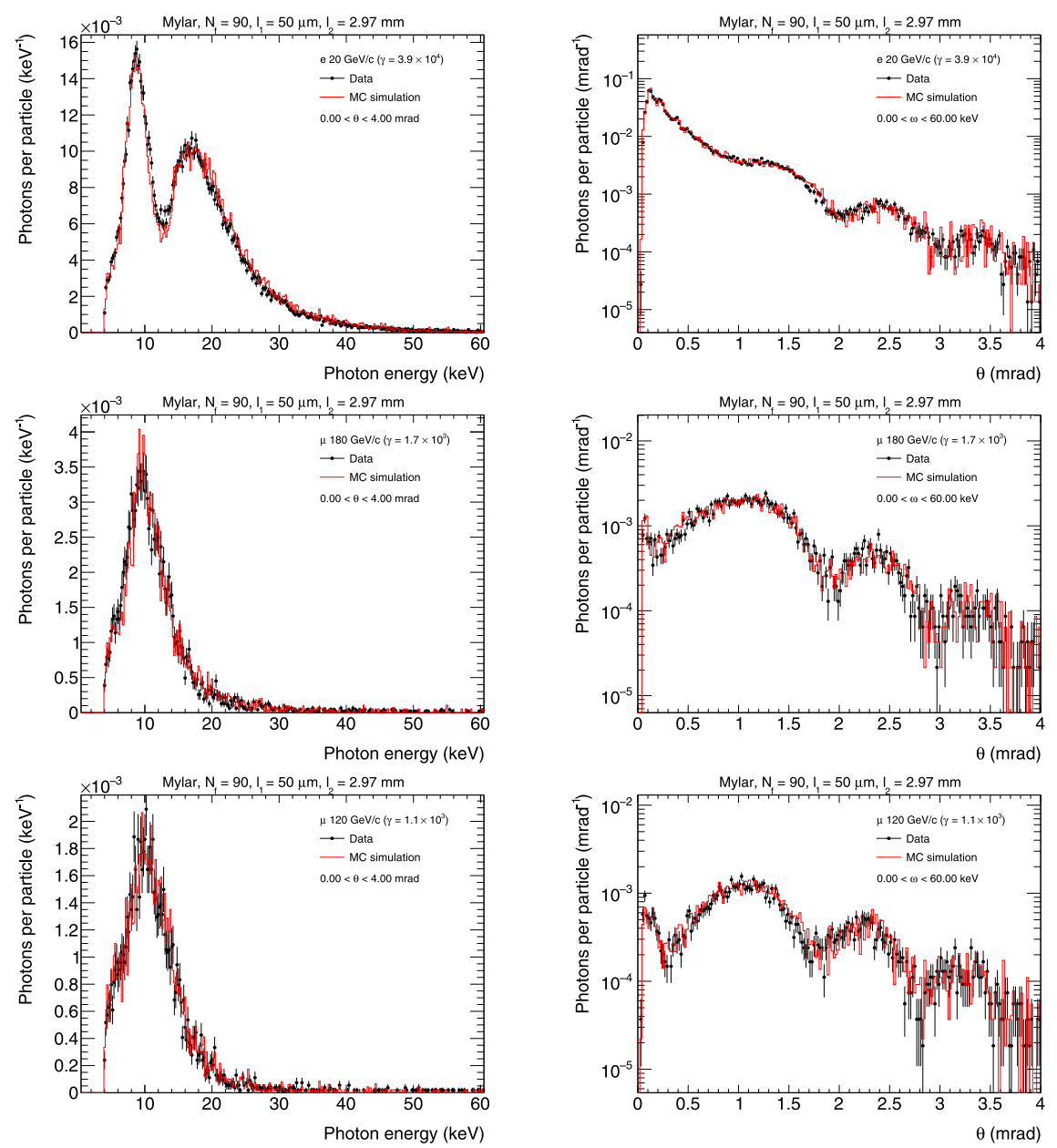

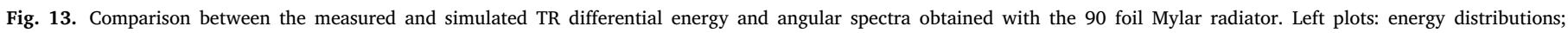

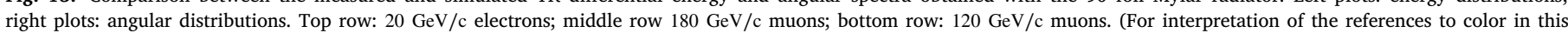
figure legend, the reader is referred to the web version of this article.)


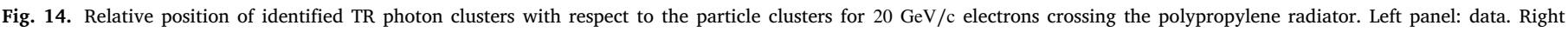
panel: MC simulation.

the last foil of each block works practically as a single foil radiator. This fact was also taken into account in the simulations. Fig. 14 shows the distribution of coordinates of the absorbed TR photons with respect to the beam particles for $20 \mathrm{GeV} / \mathrm{c}$ electrons for the polypropylene radiator. As for the mylar radiator, the distributions show generally good agreement between data and simulation results, although with some differences at small angles.

Two dimensional distributions of the photon energy versus the reconstructed production angle obtained with this radiator are shown in Fig. 15. The data are shown in the left plots and the results of the MC simulations in the right plots. The plots in the top row refer to $20 \mathrm{GeV} / \mathrm{c}$ 

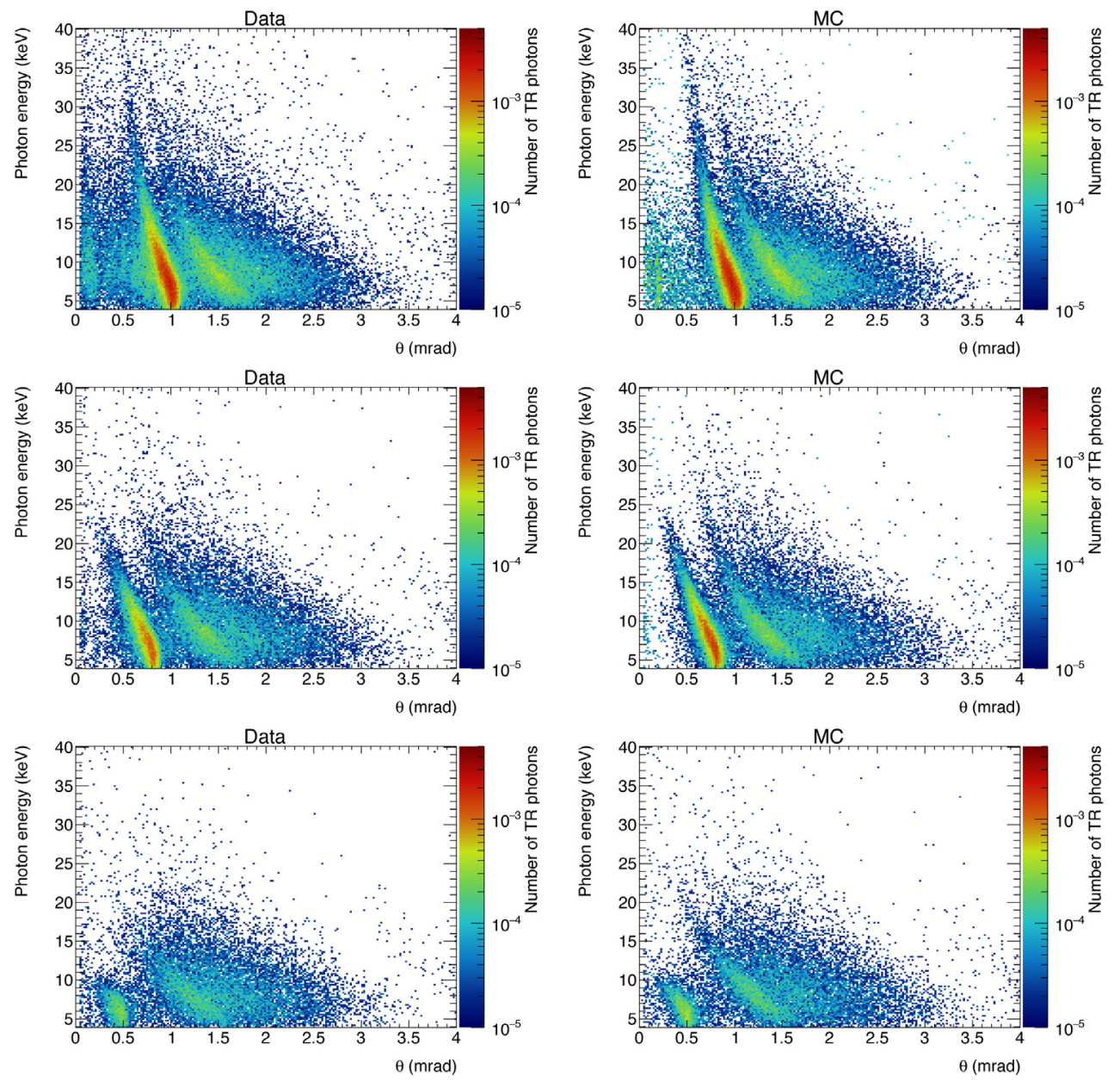

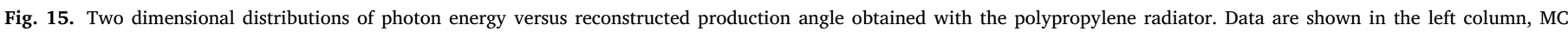

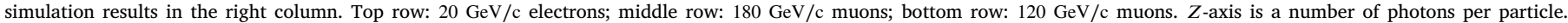

electrons, those in the middle row to $180 \mathrm{GeV} / \mathrm{c}$ muons and those in the bottom raw to $120 \mathrm{GeV} / \mathrm{c}$ muons. The most probable TR irradiation angle for $20 \mathrm{GeV} / \mathrm{c}$ electrons is near $1 \mathrm{mrad}$, and it becomes smaller for lower gamma factors. This behavior significantly deviates from the $1 / \gamma$ dependence which describes the angular distribution of the TR from a single foil. This feature is explained by interference effects, which have destructive character at small angles and push the most probable angle to higher values. The excess of clusters at angles below $0.3 \mathrm{mrad}$ is explained by the fact that the radiator has a few foils with conditions close to those of a single foil (the distance between foils of neighbor blocks is larger that the formation zone in air). Details of the energy and the angular distributions of the TR photons for the polypropylene radiator and their comparison with MC are presented in Fig. 16. The left plots show the energy distributions, while the right plots show the angular distributions. The plots in the top row refer to $20 \mathrm{GeV} / \mathrm{c}$ electrons, those in the middle row to $180 \mathrm{GeV} / \mathrm{c}$ muons and those in the bottom row to $120 \mathrm{GeV} / \mathrm{c}$ muons.

In general, data and simulation results are in a very good agreement. However some differences can be seen at small angles for electrons and in the shape of the main peak of the angular distribution for $120 \mathrm{GeV} / \mathrm{c}$ muons. These differences could be explained by mismodeling of the exact radiator structure and some underestimation of the X-ray absorption on the way to the detector sensitive volume.

The TR energy spectrum shape does not significantly change when the gamma factor changes from $\gamma \sim 3.9 \times 10^{4}$ to $\gamma \sim 1.13 \times 10^{3}$, but the number of detected photons is reduced by about a factor of 3 . The particle separation can be improved if the information on both the energy and the emission angle is used. In fact, from the twodimensional distributions of Fig. 15, one sees that selecting the region corresponding to the maximum emission for electrons (the red band at around $1 \mathrm{mrad}$ ) it is possible to suppress the contribution of particles with low gamma factors.

\subsection{Polyethylene radiator}

The polyethylene radiator was built to push the TR saturation point to a higher gamma-factor. It consists of $270 \mu \mathrm{m}$ thick foils spaced by about $3.3 \mathrm{~mm}$. The thickness of this type of polyethylene foils has large variation and the distance between foils was not accurately controlled and fluctuates up to $1 \mathrm{~mm}$. Similarly to the polypropylene radiator, a TR simulation for this radiator was done using the irregular radiator approach. In the simulation foil spacing variation of $30 \%$ was used. The angular distribution of the TR at large gamma-factors has many close peaks which cannot be resolved at our experimental conditions because of the multiple scattering effects and the smearing due to the foil spacing fluctuations. For this reason the angular spread of the TR clusters looks quite large, as shown in Figs. 17 and 18. The foil thickness affects the energy distribution of TR, which exhibits well separated peaks for electrons if no thickness fluctuations are included in the simulation. A very good quantitative agreement between experimental and simulated energy spectra was obtained with $9 \%$ variations of the foil thickness. 

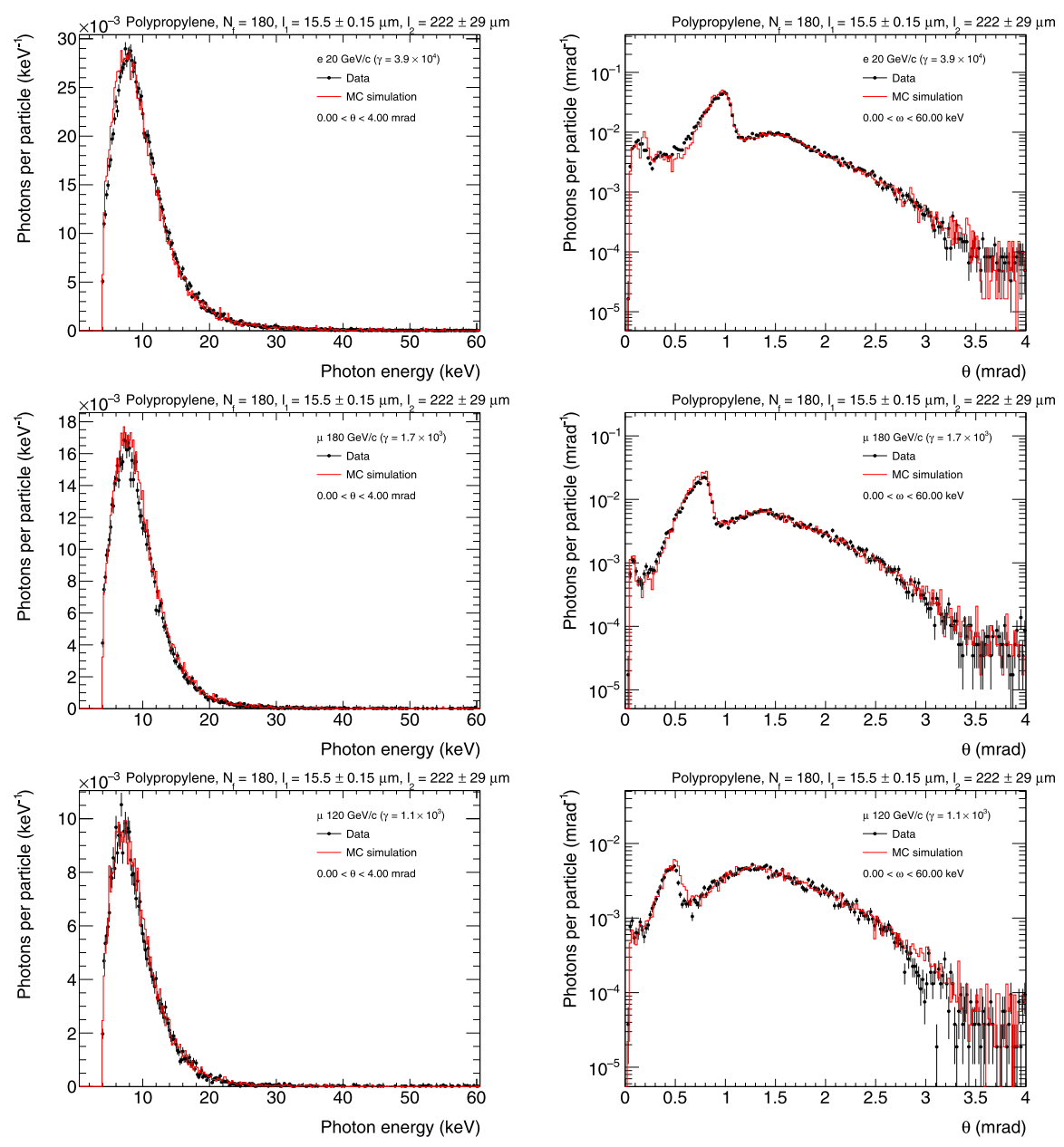

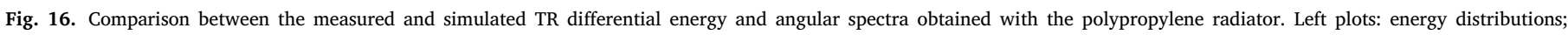

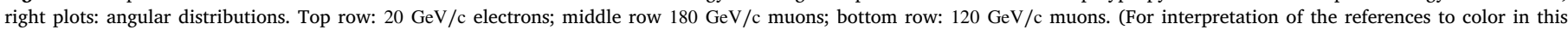
figure legend, the reader is referred to the web version of this article.)

\section{Discussion}

The information about the transition radiation production angle offers a possibility to improve the TRD particle separation power. The production angle is a complex function of the radiator parameters and cannot be evaluated with the simple law $\theta=1 / \gamma$. An estimate of the angular position of interference maxima can be obtained using the formula [36]:

$\theta=\sqrt{\frac{4 \pi^{2} c^{2} r^{2}}{\left(l_{1} \omega_{1}^{2}+l_{2} \omega_{2}^{2}\right)\left(l_{1}+l_{2}\right)}-1 / \gamma^{2}}$.

For the polyethylene radiator with $l_{1} \ll l_{2}$ and $\omega_{1} \gg \omega_{2}$ the estimate for the angle of the first interference maximum $(r=1)$ is given by

$\theta \approx \sqrt{\frac{4 \pi^{2} c^{2}}{l_{1} l_{2} \omega_{1}^{2}}-1 / \gamma^{2}}$

taking $\gamma_{\text {sat }} \approx 0.6 \omega_{1} \sqrt{\ell_{1} \ell_{2}} / c$

$\theta \approx \sqrt{1.4 \pi^{2} / \gamma_{\text {sat }}^{2}-1 / \gamma^{2}}$.

This angle corresponds to the last interference maximum of the energy spectra where most of the TR energy is emitted, i.e. the region around $\omega_{\max }=\ell_{1} \omega_{1}^{2} / 2 \pi c$. A few important conclusions can be drawn from this formula. For the defined radiator parameters, the higher is the gamma-factor, the larger is the angle of the first interference maximum. It reaches almost its asymptotic limit $\theta_{\max }$ at $\gamma=\gamma_{\text {sat }}$. For $\gamma / \gamma_{\text {sat }}=1 / 3$ the angle is about $0.6 \theta_{\max }$. This fact can be used to enhance the separation of particles with close gamma factors. The increase of the $\gamma_{\text {sat }}$ value leads to a decrease of the main TR production angle, Eq. (12).

The simplified numerical expressions can be used for practical estimation of the main TR production parameters: $\theta \sim 1.2 / \omega_{1} \sqrt{l_{1} l_{2}}$, $\gamma_{\text {thr }} \sim 3 \times 10^{3} \omega_{1} \ell_{1}, \gamma_{\text {sat }} \sim 3 \times 10^{3} \omega_{1} \sqrt{\ell_{1} \ell_{2}}$ and $\omega_{\max }=0.65 \ell_{1} \omega_{1}^{2}$, where $\theta$ in $\mathrm{mrad}, \omega_{1}$ in $\mathrm{eV}, \omega_{\max }$ in $\mathrm{keV}$ and $l_{1}$ and $l_{2}$ in $\mathrm{mm}$.

The detector sensor material is a very important parameter of the TRD optimization. A $500 \mu \mathrm{m}$ thick silicon detector has sufficient X-ray absorption power up to $20 \mathrm{keV}$ (40\% absorption efficiency) and can be very efficient in a combination with radiators with relatively small foil thickness. This type of radiators produces rather soft TR photons at quite large angles. This feature allows to reduce the distance between the pixel detector and the radiator down to $0.5 \mathrm{~m}$ or even less. Such detectors can be effectively used for electron-pion separation as a part of precision tracking systems.

The higher $\gamma_{\text {sat }}$ the higher is the most probable TR photon energy. Silicon sensors with larger thicknesses (up to $1 \mathrm{~mm}$ ) are also available, but for high TR energies the most promising sensor material is GaAs. In fact, a $500 \mu \mathrm{m}$ thick GaAs sensor has $40 \%$ absorption efficiency at a photon energy of $60 \mathrm{keV}$; in addition, GaAs sensors can be produced with thicknesses up to $2 \mathrm{~mm}$. First results of TR studies with this type of detectors are published in [37]. Detailed results of TR studies with GaAs detector will be published elsewhere. 

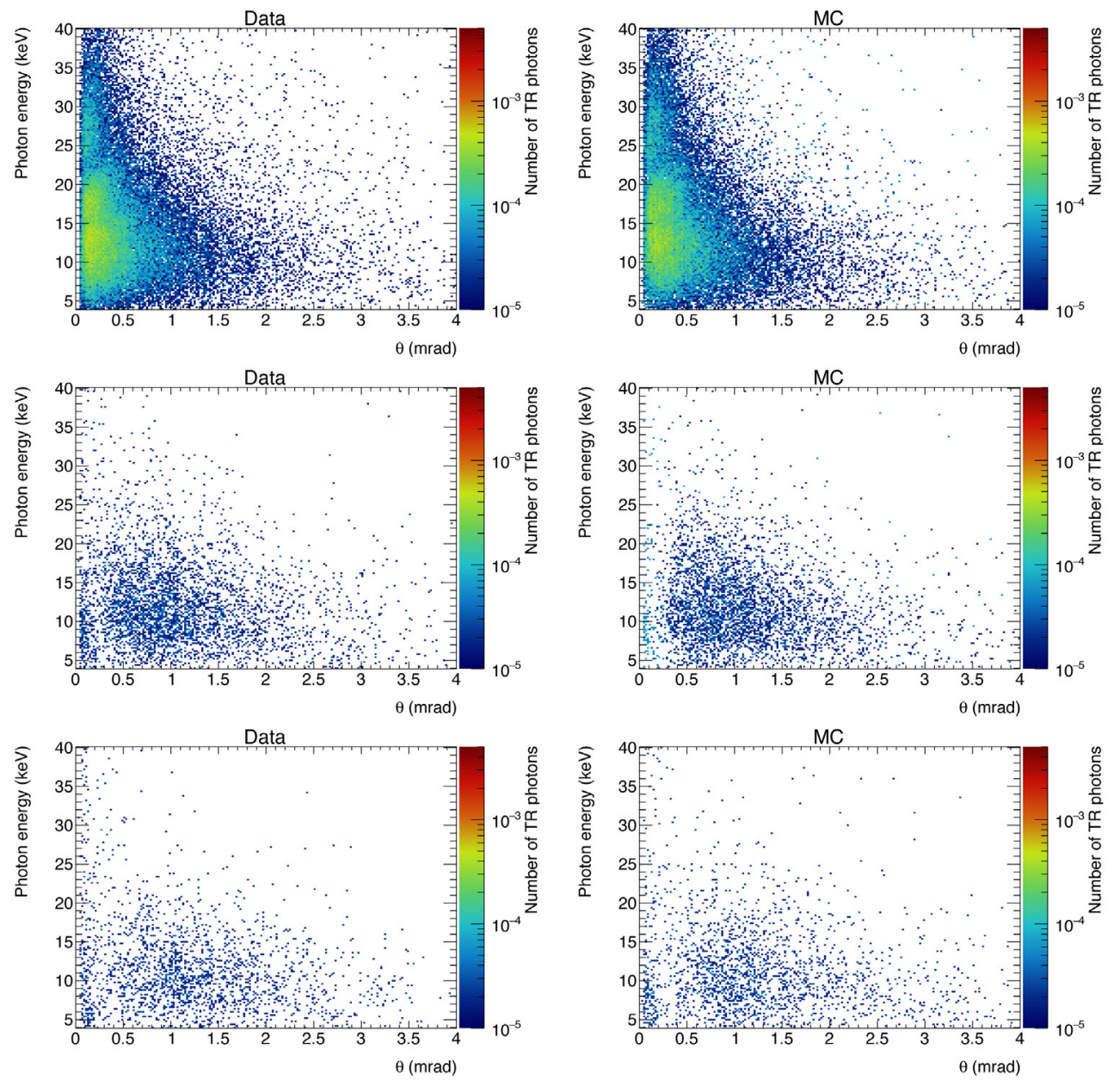

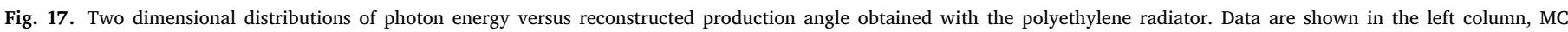

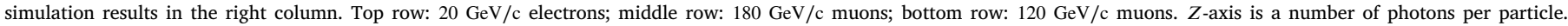

\section{Conclusions}

The excellent spatial and energy resolutions of a semiconductor sensor bump bonded to a Timepix3 chip has offered the unique opportunity for precise simultaneous measurements of spectral and angular parameters of transition radiation photons. A $480 \mu \mathrm{m}$ thick silicon sensor attached to the Timepix 3 chip was exposed to pion, electron and muon beams of different energies at the CERN SPS. High resolution images of the energy - angle phase space of the TR produced by different radiators were obtained and compared with MC simulations. The characteristic interference patterns are in agreement with the theoretical models with an unprecedented level of details. The studies presented in this paper also show that simultaneous measurements of both the energy and the emission angles of the TR X-rays could be used to enhance the particle identification performances of TRDs.

Interference effects between foils change the angular distribution of the TR. It was shown that the commonly used law $\theta \sim 1 / \gamma$ is not applicable for the estimate of the main TR emission angle. For instance, for $20 \mathrm{GeV} / \mathrm{c}$ electrons crossing the polypropylene radiator consisting of $15.5 \mu \mathrm{m}$ foils spaced by $222 \mu \mathrm{m}$ the most probable TR emission angle is about $1 \mathrm{mrad}$ instead of $0.025 \mathrm{mrad}$, as would be expected from the $1 / \gamma$ law. This fact can be used to design compact TRDs for electron-pion separation. It was also shown that different parts of the TR energy and angular spectra have different $\gamma$ - dependencies. This allows to sharpen the dependence of the TR production on the Lorentz factor and can be used for the separation of particles with close gamma factors.

\section{Declaration of competing interest}

The authors declare that they have no known competing financial interests or personal relationships that could have appeared to influence the work reported in this paper.

\section{CRediT authorship contribution statement}

J. Alozy: Resources, Methodology. N. Belyaev: Software, Formal analysis, Investigation, Writing - review \& editing, Visualization. M. Campbell: Resources, Methodology. M. Cherry: Conceptualization, Formal analysis. F. Dachs: Resources, Methodology, Investigation, Writing - original draft. S. Doronin: Resources, Investigation. K. Filippov: Resources, Investigation. P. Fusco: Resources, Investigation. F. Gargano: Resources, Investigation. E.H.M. Heijne: Resources, Methodology. S. Konovalov: Resources, Investigation. D. Krasnopevtsev: Resources, Investigation. X. Llopart: Resources, Methodology. F. Loparco: Investigation, Formal analysis, Software, Writing - review \& editing. V. Mascagna: Resources, Investigation. M.N. Mazziotta: Investigation, Formal analysis. H. Pernegger: Resources, Methodology. D. Ponomarenko: Data curation, Investigation, Visualization. C. Rembser: Resources, Investigation. A. Romaniouk: Conceptualization, Methodology, Formal analysis, Investigation, Supervision, Writing - original draft, Project administration, Funding acquisition. A.A. Savchenko: Formal analysis, Investigation, Software. D. Schaefer: Resources, Methodology, Software. E.J. Schioppa: Resources, Methodology, Software, Formal analysis, Visualization, Writing - original draft. 

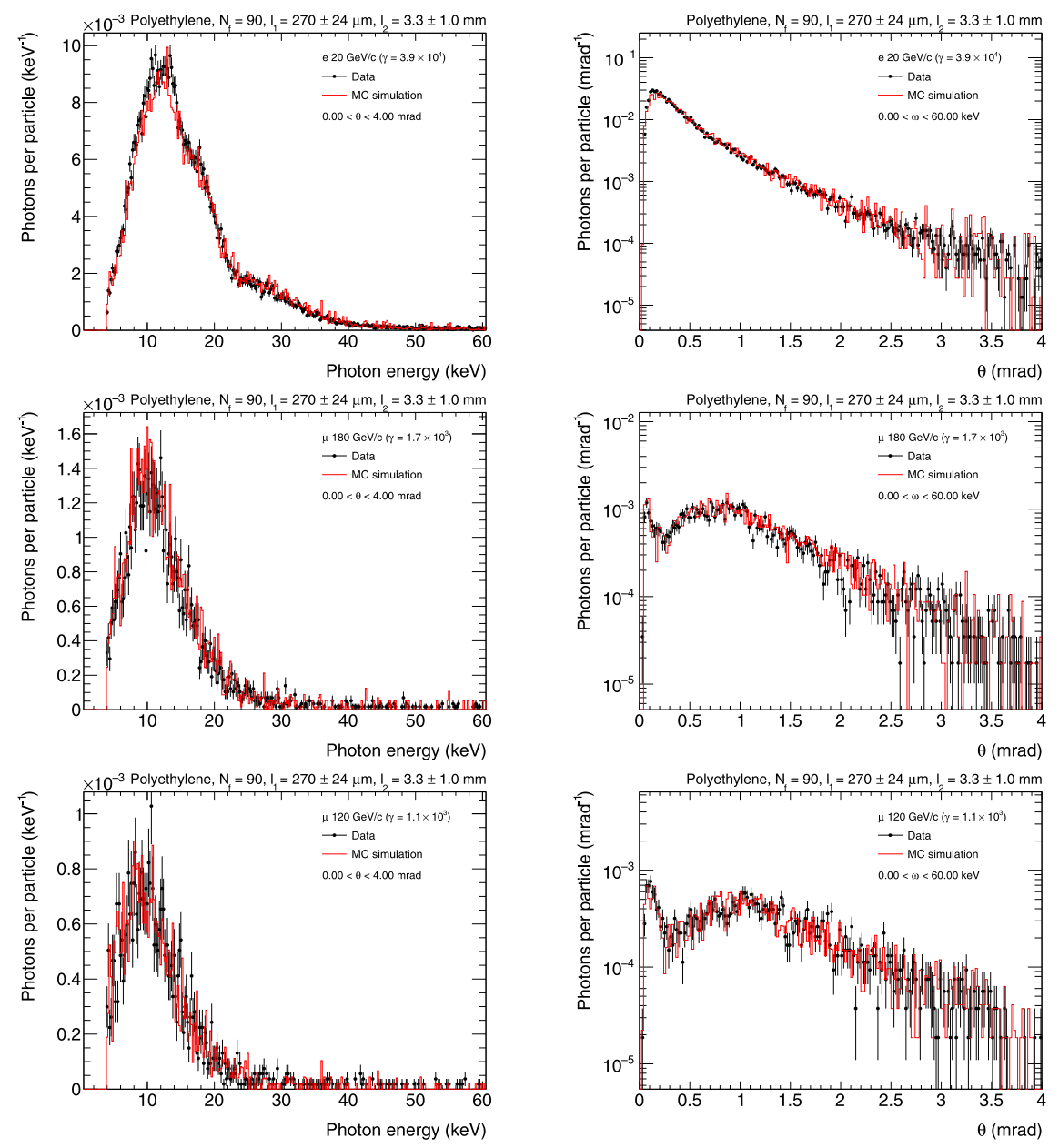

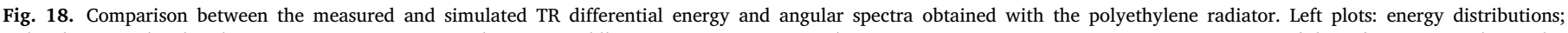

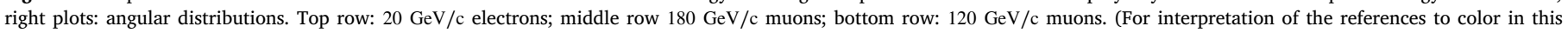
figure legend, the reader is referred to the web version of this article.)

D.Yu. Sergeeva: Formal analysis, Visualization. E. Shulga: Software, Formal analysis, Investigation. S. Smirnov: Software, Formal analysis, Project administration, Funding acquisition. Y. Smirnov: Software, Formal analysis, Investigation, Visualization. M. Soldani: Resources, Investigation. P. Spinelli: Conceptualization, Formal analysis. M. Strikhanov: Funding acquisition. P. Teterin: Resources, Investigation. V. Tikhomirov: Software, Formal analysis, Investigation. A.A. Tishchenko: Formal analysis, Visualization. M. van Beuzekom: Resources, Methodology, Software, Visualization. B. van der Heijden: Resources, Methodology. K. Vorobev: Resources, Investigation. K. Zhukov: Resources, Investigation.

\section{Acknowledgment}

We gratefully acknowledge the financial support from Russian Science Foundation grant (project No. 16-12-10277).

\section{References}

[1] G.M. Garibian, Contribution to the theory of transition radiation, Sov. Phys.-JETP 6 (1958) 1079.

[2] G.M. Garibian, Transition radiation effects in particle energy losses, Sov. Phys._JETP 10 (1960) 372.

[3] X. Artru, G.B. Yodh, G. Mennessier, Practical theory of the multilayered transition radiation detector, Phys. Rev. D12 (1975) 1289, http://dx.doi.org/10.1103/ PhysRevD.12.1289.
[4] C. Patrignani, et al., Particle Data Group Collaboration, Review of particle physics, Chin. J. Phys C40 (10) (2016) 100001, http://dx.doi.org/10.1088/16741137/40/10/100001.

[5] B. Dolgoshein, Transition radiation detectors, Nucl. Instrum. Methods A326 (1993) 434-469, http://dx.doi.org/10.1016/0168-9002(93)90846-A.

[6] A. Andronic, J.P. Wessels, Transition radiation detectors, Nucl. Instrum. Methods A666 (2012) 130-147, http://dx.doi.org/10.1016/j.nima.2011.09.041.

[7] C.W. Fabjan, et al., Practical prototype of a cluster counting transition radiation detector, Nucl. Instrum. Methods 185 (1981) 119, http://dx.doi.org/10.1016/ 0029-554X(81)91202-7.

[8] V. Commichau, M. Deutschmann, H. Goddeke, K. Hangarter, U. Putzhofen, R. Schulte, W. Struczinski, A transition radiation detector for pion identification in the $100 \mathrm{GeV} / \mathrm{c}$ momentum region, Nucl. Instrum. Methods 176 (1980) 325-331, http://dx.doi.org/10.1016/0029-554X(80)90724-7.

[9] M. Deutschmann, et al., Particle identification using the angular distribution of transition radiation, Nucl. Instrum. Methods 180 (1981) 409, http://dx.doi.org/ 10.1016/0029-554X(81)90080-X.

[10] T. Ludlam, et al., Particle identification by electron cluster detection of transition radiation photons, Nucl. Instrum. Methods 180 (1981) 413, http://dx.doi.org/10. 1016/0029-554X(81)90081-1.

[11] M. Albrow, A very forward hadron spectrometer for the LHC and cosmic ray physics, in: Proceedings, 2nd World Summit on Exploring the Dark Side of the Universe (EDSU2018): Point a Pitre, Guadeloupe, France, June 25-29, 2018, PoS EDSU2018 (2018) 048, http://dx.doi.org/10.22323/1.335.0048.

[12] G.L. Case, P.P. Altice, M.L. Cherry, J. Isbert, J.W. Mitchell, D. Patterson, Measurements of compton scattered transition radiation at high Lorentz factors, Nucl. Instrum. Methods A524 (2004) 257-263, http://dx.doi.org/10.1016/j. nima.2004.01.066, arXiv:hep-ex/0209038.

[13] S.P. Wakely, D. Muller, F. Gahbauer, S. Plewnia, J.R. Horandel, Transition radiation detectors for energy measurements at high Lorentz factors, in: Proceedings, 5th International Workshop on Radiation Imaging Detectors (IWORID 
2003): Riga, Latvia, September 7-11, 2003, Nucl. Instrum. Methods A531 (2004) 435-444, http://dx.doi.org/10.1016/j.nima.2004.04.245.

[14] N. Belyaev, et al., Test beam studies of possibilities to separate particles with gamma factors above $10^{3}$ with straw based transition radiation detector, in: Proceedings, 3rd International Conference on Particle Physics and Astrophysics (ICPPA 2017): Moscow, Russia, October 2-5, 2017, J. Phys. Conf. Ser. 934 (1) (2017) 012053, http://dx.doi.org/10.1088/1742-6596/934/1/012053.

[15] N. Belyaev, et al., Development of transition radiation detectors for hadron identification at TeV energy scale, J. Phys. Conf. Ser. (2017) in print.

[16] K.A. Ispirian, R.K. Ispirian, A.T. Margarian, X-ray transition radiation detectors (XTRD) for $\mathrm{e} / \mathrm{pi}$ and $\mathrm{pi} / \mathrm{k} / \mathrm{p}$ identification in $\mathrm{TeV}$ energy region, Nucl. Instrum. Methods A336 (1993) 533-541, http://dx.doi.org/10.1016/01689002(93)91261-K.

[17] K.K. Shikhlyarov, N.Z. Akopov, V.G. Gavalian, X-ray transition radiation detector (XTRD) for hadron identification in high-energy beams, Nucl. Instrum. Methods A398 (1997) 189-194, http://dx.doi.org/10.1016/S0168-9002(97)00768-7.

[18] M.L. Cherry, Measuring the lorentz factors of energetic particles with transition radiation, in: Proceedings, TRDs for the 3rd Millenium: 4th Workshop on Advanced Transition Radiation Detectors for Accelerator and Space Applications (TRD2011): Bari, Italy, September 14-16, 2011, Nucl. Instrum. Methods A706 (2013) 39-42, http://dx.doi.org/10.1016/j.nima.2012.05.008.

[19] N. Belyaev, et al., Measurements of angular distribution and spectrum of transition radiation with a GridPix detector, in: Proceedings, 3rd International Conference on Particle Physics and Astrophysics (ICPPA 2017): Moscow, Russia, October 2-5, 2017, J. Phys. Conf. Ser. 934 (1) (2017) 012049, http://dx.doi. org/10.1088/1742-6596/934/1/012049.

[20] S. Furletov, J. Furletova, Geant4 simulation of transition radiation detector based on DEPFET silicon pixel matrices, in: Proceedings, TRDs for the 3rd Millenium: 4th Workshop on Advanced Transition Radiation Detectors for Accelerator and Space Applications (TRD2011): Bari, Italy, September 14-16, 2011, Nucl. Instrum. Methods A706 (2013) 73-78, http://dx.doi.org/10.1016/j.nima.2012. 05.009.

[21] J. Alozy, et al., Identification of particles with lorentz factor up to $10^{4}$ with transition radiation detectors based on micro-strip silicon detectors, Nucl. Instrum. Methods A927 (2019) 1-13, http://dx.doi.org/10.1016/j.nima.2019.02. 032, arXiv:1901.11265.

[22] R. Ballabriga, M. Campbell, X. Llopart, Asic developments for radiation imaging applications: The medipix and timepix family, Nucl. Instrum. Methods A878 (2018) 10-23, http://dx.doi.org/10.1016/j.nima.2017.07.029.

[23] E.J. Schioppa, et al., First measurements of the spectral and angular distribution of transition radiation using a silicon pixel sensor on a Timepix3 chip, in: 14th Pisa Meeting on Advanced Detectors: Frontier Detectors for Frontier Physics (Pisameet) la Biodola-Isola D'Elba, Livorno, Italy, May 27-June 2, 2018, Nucl. Instrum. Methods A936 (2019) 523-526, http://dx.doi.org/10.1016/j.nima.2018. 11.062 .
[24] X. Llopart, R. Ballabriga, M. Campbell, L. Tlustos, W. Wong, Timepix, a 65k programmable pixel readout chip for arrival time, energy and/or photon counting measurements, in: Instrumentation. Proceedings, 11th International Conference, VCI 11, Vienna, Austria, February 19-24, 2007, Nucl. Instrum. Methods A581 (2007) 485-494, http://dx.doi.org/10.1016/j.nima.2007.08.079,10.1016/j.nima. 2007.11.003.

[25] T. Poikela, et al., Timepix3: a 65K channel hybrid pixel readout chip with simultaneous ToA/ToT and sparse readout, J. Instrum. 9 (05) (2014) C05013, http: //dx.doi.org/10.1088/1748-0221/9/05/c05013, URL https://doi.org/10.1088/ $1748-0221 / 9 / 05 / c 05013$.

[26] J. Jakubek, Precise energy calibration of pixel detector working in time-overthreshold mode, in: Proceedings, 13th International Workshop on Radiation Imaging Detectors (IWORID2011): Villigen and Zurich, Switzerland, July 3-7, 2011, Nucl. Instrum. Methods A633 (2011) S262-S266, http://dx.doi.org/10. 1016/j.nima.2010.06.183.

[27] D.M. Saunders, LHCb Collaboration Collaboration, The Timepix3 telescope for LHCb upgrade RD measurements, in: Proceedings, 38th International Conference on High Energy Physics (ICHEP 2016): Chicago, IL, USA, August 3-10, 2016, PoS ICHEP2016 (2017) 1186, http://dx.doi.org/10.22323/1.282.1186.

[28] K. Akiba, et al., LHCb VELO Timepix3 telescope, J. Instrum. 14 (05) (2019) P05026, http://dx.doi.org/10.1088/1748-0221/14/05/P05026, arXiv: 1902.09755 .

[29] B. van der Heijden, J. Visser, M. van Beuzekom, H. Boterenbrood, S. Kulis, B. Munneke, F. Schreuder, SPIDR, a general-purpose readout system for pixel ASICs, in: Proceedings, Topical Workshop on Electronics for Particle Physics (TWEPP 2016): Karlsruhe, Germany, J. Instrum. 12 (02) (2017) C02040, http: //dx.doi.org/10.1088/1748-0221/12/02/C02040.

[30] M. Kiehn, et al., Proteus beam telescope reconstruction, 2019, http://dx.doi.org/ 10.5281/zenodo.2579153.

[31] K. Akiba, et al., Charged particle tracking with the Timepix ASIC, Nucl. Instrum. Methods A661 (2012) 31-49, http://dx.doi.org/10.1016/j.nima.2011. 09.021, arXiv:1103.2739.

[32] M.L. Cherry, G. Hartmann, D. Muller, T.A. Prince, Transition radiation from relativistic electrons in periodic radiators, Phys. Rev. D10 (1974) 3594-3607, http://dx.doi.org/10.1103/PhysRevD.10.3594.

[33] G.M. Garibian, L.A. Gevorgian, C. Yang, The calculation of x-ray transition radiation generated in regular and irregular-layered media, Nucl. Instrum. Methods 125 (1975) 133-137, http://dx.doi.org/10.1016/0029-554X(75)90565-0.

[34] https://www.nist.gov/pml/x-ray-mass-attenuation-coefficients.

[35] E.J. Schioppa, J. Idarraga, M. van Beuzekom, J. Visser, E. Koffeman, E. Heijne, K.J. Engel, J. Uher, Study of charge diffusion in a silicon detector using an energy sensitive pixel readout chip, IEEE Trans. Nucl. Sci. 62 (2015) 2349-2359, http://dx.doi.org/10.1109/TNS.2015.2475124.

[36] A.A. Savchenko, D.Y. Sergeeva, A.A. Tishchenko, M.N. Strikhanov, Small-angle X-ray transition radiation from multilayered structures, Phys. Rev. D99 (1) (2019) 016015, http://dx.doi.org/10.1103/PhysRevD.99.016015.

[37] F. Dachs, et al., Transition radiation measurements with a silicon and a gallium arsenide pixel sensor on a Timepix3 chip, Nucl. Instrum. Methods A958 (2020) 162037, http://dx.doi.org/10.1016/j.nima.2019.03.092, in press. 